\title{
LOCALLY GEODESICALLY QUASICONVEX FUNCTIONS ON COMPLETE RIEMANNIAN MANIFOLDS ${ }^{1}$
}

\author{
TAKAO YAMAGUCHI
}

\begin{abstract}
In this article, we investigate the topological structure of complete Riemannian manifolds admitting locally geodesically quasiconvex functions, whose family includes all geodesically convex functions. The existence of a locally geodesically quasiconvex function is equivalent to the existence of a certain filtration by locally convex sets. Our argument contains Morse theory for the lower limit function of a given locally geodesically quasiconvex function.
\end{abstract}

0. Introduction. A function on a Riemannian manifold is geodesically convex if its restriction to every geodesic segment is convex as one variable function in the usual sense. Geodesically convex functions arise naturally in a number of geometric contexts, and the topological and Riemannian structures of complete manifolds which admit certain geodesically convex functions have been investigated $[\mathbf{1}, \mathbf{7}$ 10, 14]. Specifically, a recent result of Greene and Shiohama [9] states that if a complete Riemannian manifold $M$ admits a locally nonconstant geodesically convex function $\varphi: M \rightarrow R$ without minimum and with $\{x \in M ; \varphi(x)=a\}$ compact for all $a>\inf _{M} \varphi$ and if $M$ has noncompact isometry group, then $M$ is isometric to the Riemannian product of a compact Riemannian manifold and the real line. A function is called locally nonconstant if it is nonconstant on every nonempty open subset. This result shows that the existence of geodesically convex functions of certain types imposes strong restrictions on the Riemannian structure of the manifolds. On the other hand, it is known $[2,6]$ that the inclusion map of a closed totally convex set in a complete manifold (it appears, for instance, as a sublevel set of a geodesically convex function) into the whole manifold is a homotopy equivalence. A subset of a Riemannian manifold is called totally convex if it includes all geodesic segments whose end points lie in it. ${ }^{1}$

From this point of view, it is natural to expect that the topological structure of manifolds, for instance in the sense of homeomorphism or diffeomorphism type, may be restricted by the existence of more general functions than geodesically convex functions. We say that a function $\varphi: M \rightarrow R \cup\{-\infty\}$ is geodesically quasiconvex on an open subset $U$ of $M$ if its restriction to every geodesic segment $\gamma:[a, b] \rightarrow U$ is quasiconvex, that is,

$$
\varphi \circ \gamma(t) \leq \max \{\varphi \circ \gamma(a), \varphi \circ \gamma(b)\}
$$

for all $t \in[a, b]$. A function $\varphi$ is geodesically strictly quasiconvex on $U$ if the inequality is strict whenever $t \in(a, b)$ and $\gamma$ is nonconstant. If each point in $M$ has

\footnotetext{
Received by the editors November 16, 1984 and, in revised form, August 2, 1985.

1980 Mathematics Subject Classification. Primary 53C20, 53C22.

${ }^{1}$ Research partially supported by the Japan Society for the Promotion of Science and by the Institute of Mathematics, University of Tsukuba.
} 
a neighborhood on which $\varphi$ is geodesically quasiconvex (resp. geodesically strictly quasiconvex), then $\varphi$ is locally geodesically quasiconvex (resp. locally geodesically strictly quasiconvex). If $\varphi$ is geodesically quasiconvex on $M$ (resp. geodesically strictly quasiconvex on $M$ ), then it is simply said to be geodesically quasiconvex (resp. geodesically strictly quasiconvex). We abbreviate "geodesically convex" and "geodesically quasiconvex" as "convex" and "quasiconvex", etc.

It is well known that convex functions are locally Lipschitz continuous, and therefore differentiable almost everywhere. On the other hand, locally quasiconvex functions are not necessarily continuous. It should also be remarked that there are numerous complete manifolds which admit locally nonconstant quasiconvex functions, but not locally nonconstant convex functions. We shall give an example of a Riemannian manifold with this property (Example 3 in $§ 1$ ).

There is one metric consequence of the existence of a certain locally quasiconvex function on a complete manifold: The volume of such a manifold is infinite (Proposition 3.4). The main purpose of the present paper is to investigate the topological structure of complete manifolds admitting locally nonconstant locally quasiconvex functions. We note that every Riemannian manifold admits a continuous nonconstant locally quasiconvex function which is constant on an open set (see Example 1). Hence, to obtain a topological implication of the manifold, it is natural to consider only locally nonconstant locally quasiconvex functions.

Let $\varphi: M \rightarrow R \cup\{-\infty\}$ be a locally nonconstant locally quasiconvex function on a complete Riemannian manifold $M$ of dimension $n$. We denote by $M^{a}(\varphi)$ the sublevel set $\{x \in M ; \varphi(x) \leq a\}$ for the sake of brevity, and note that $M^{a}(\varphi)$ is locally convex (for the definition, see $\S 1$ ). It follows from a result of Cheeger and Gromoll [6] that each connected component of the closure $\overline{M^{a}(\varphi)}$ of $M^{a}(\varphi)$ has manifold structure with (possibly empty) boundary $\partial M^{a}(\varphi)$ for every $a \in \varphi(M)$. If $M^{a}(\varphi)$ has a connected component of dimension $n$, then it is connected (Lemma 3.3). We consider the lower limit function $\varphi^{*}: M \rightarrow R \cup\{-\infty\}$ of $\varphi$ defined by $\varphi^{*}(p)=\inf \left\{a ; p \in \overline{M^{a}(\varphi)}\right\}$, and note that $\varphi$ is lower semicontinuous if and only if $\varphi=\varphi^{*}$, and that $\varphi^{*}$ is also locally quasiconvex. The function $\varphi^{*}$ is well suited to our purpose. We set

$$
\lambda_{1}=\inf \left\{a ; \operatorname{dim} M^{a}(\varphi)=n\right\}, \quad M_{\varphi}=M^{\lambda_{1}}\left(\varphi^{*}\right) .
$$

The set $M_{\varphi}$ is a closed connected locally convex set with $\operatorname{dim} M_{\varphi}<n$ if it is nonempty (Lemmas 3.8 and 4.2). It is just the minimum set, when $\varphi$ is continuous.

We shall prove the following topological structure theorems.

THEOREM A. Suppose that $M_{\varphi}$ is nonempty. Then:

(1) There is a smooth manifold $L$ such that $M-M_{\varphi}$ is diffeomorphic to $L \times R$.

(2) $M$ is homeomorphic to the normal bundle $\nu\left(M_{\varphi}\right)$ of $M_{\varphi}$ in $M$. If $M_{\varphi}$ has no boundary, then $M$ is diffeomorphic to $\nu\left(M_{\varphi}\right)$.

COROLLARY. If $M$ admits a lower semicontinuous locally strictly quasiconvex function $\varphi$ with a minimum such that $\operatorname{dim} M^{a}(\varphi)=n$ for every $a \in \varphi(M), a>$ $\inf _{M} \varphi$, then $M$ is diffeomorphic to Euclidean space $R^{n}$.

In fact, $\varphi$ is of course locally nonconstant, and $M_{\varphi}$ coincides with the minimum set of $\varphi$, which must consist of a single point, because it is connected and locally convex.

In some special cases, $M$ has a product structure. 
THEOREM B. Suppose that for some $a \in \varphi(M)$ with $\operatorname{dim} M^{a}(\varphi)=n, \partial M^{a}(\varphi)$ is disconnected and has a compact connected component. Then:

(1) $\varphi^{*}$ has a minimum.

(2) $M_{\varphi}$ is a compact totally convex hypersurface of $M$ without boundary, and the normal bundle of $M_{\varphi}$ in $M$ is trivial.

(3) Each compact connected component of $\partial M^{a}(\varphi)$ is homeomorphic to $M_{\varphi}$, and $M$ is diffeomorphic to $M_{\varphi} \times R$.

THEOREM C. Suppose that $\varphi^{*}$ has no minimum and that for some $a \in \varphi(M)$ with $\operatorname{dim} M^{a}(\varphi)=n, \partial M^{a}(\varphi)$ has a compact connected component. Then:

(1) $M^{c}(\varphi)$ has dimension $n$ and $\partial M^{c}(\varphi)$ is connected and compact for every $c \in\left(\inf _{M} \varphi, a\right]$. In particular $M_{\varphi}$ is empty.

(2) There is a smooth compact manifold $L$ homeomorphic to $\partial M^{a}(\varphi)$ such that $M$ is diffeomorphic to $L \times R$.

In the case where $M_{\varphi}$ is empty and all the connected components of $\partial M^{a}(\varphi)$ are noncompact for every $a \in \varphi(M)$, it is unknown whether $M$ has a topological product structure even if $\varphi$ is continuous. We shall construct such a function $\varphi$ on the Poncaré disk $M^{2}$ such that $\partial M^{a}(\varphi)$ consists of infinitely many noncompact connected components for every $a \in \varphi(M)$ (Example 9). The end structure, however, can be completely described:

THEOREM D. If $M$ admits a locally nonconstant locally quasiconvex function, then $M$ has at most two erds.

For the sake of intuitive comprehensibility, we give some examples of (locally) quasiconvex functions in $\S 1$. After some preliminary arguments in $\S \S 2$ and 3 , the reduction of $\varphi$ to $\varphi^{*}$ is carried out in $\S 4$. The main technique for the proofs is to construct a smooth complete vector field $X$ over $M$ associated with $\varphi: M \rightarrow$ $R \cup\{-\infty\}, a>\lambda_{1}$ and a compact set $K$ in Int $M^{a}(\varphi)$ with the following properties: The function $\varphi^{*}$ and the distance functions from all points in $K$ are strictly decreasing along each integral curve of $X$ within $M$ - Int $M^{a}(\varphi)$, and the integral curves of $X$ intersect sublevel sets of $\varphi^{*}$ transversally at all points in $M-M^{a}(\varphi)$. If the vector field $X$ were constructed, the Morse theoretic argument would apply to the lower semicontinuous function $\varphi^{*}$. This is achieved in $\S 5$. $\S \S 6$ and 7 are devoted to prove the topological structure theorems. The end structure is discussed in $\S 8$.

For a point $p$ and a subset $S$ in $M$, and for $\varepsilon>0$, we denote by $B(p, \varepsilon)$ and $T_{\varepsilon}(S)$ the metric ball of radius $\varepsilon$ around $p$ and the $\varepsilon$-neighborhood of $S$ in $M$ respectively.

Quasiconvex functions are closely related to peakless functions. For information about the basic properties of peakless functions, see Busemann and Phadke [5].

ACKNOWLEDGMENT. The author would like to express his thanks to Professor $\mathrm{K}$. Shiohama for valuable advice and constant encouragements. He is also indebted to Professor A. Morimoto for kind advice on the first manuscript.

1. Examples. Let $M$ be a (not necessarily complete) Riemannian manifold. A subset $A$ of $M$ is called convex if any two points in $A$ are joined by a unique minimizing geodesic segment, and if it lies in $A$. A subset $A$ of $M$ is called locally convex if any point in $\bar{A}$ has a neighborhood $U$ in $M$ such that $U \cap A$ is convex. 


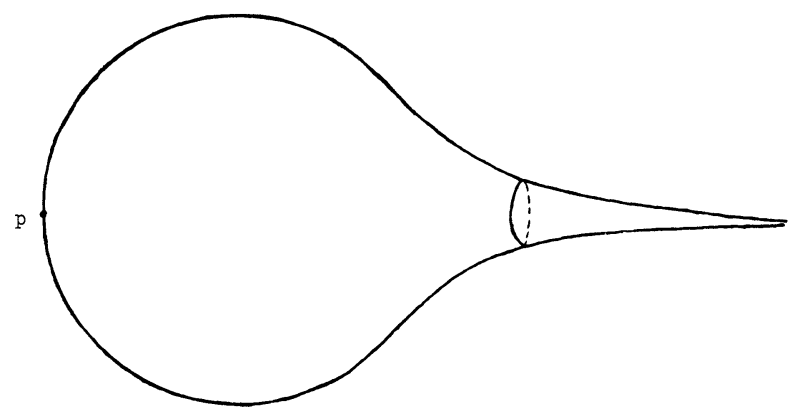

FIGURE 1

In this section, we give some examples of (locally) quasiconvex functions, which will be useful in later arguments. First of all, we note that a function $\varphi: M \rightarrow$ $R \cup\{-\infty\}$ is quasiconvex if and only if $M^{a}(\varphi)$ is totally convex for every $a \in \varphi(M)$, and that $\varphi$ is locally quasiconvex if and only if every point in $M$ has a neighborhood $U$ such that $M^{a}(\varphi) \cap U$ is convex whenever it is nonempty. We denote by $d$ the distance function induced from the Riemannian metric on $M$.

EXAMPLE 1. Let $M$ be an arbitrary Riemannian manifold. For any point $p$ in $M$, take a positive number $r$ such that $B(p, \rho)$ is convex for $0 \leq \rho \leq r$. The function $\varphi: M \rightarrow R$ defined by

$$
\varphi(q)= \begin{cases}d(p, q) & \text { if } d(p, q)<r \\ r & \text { if } d(p, q) \geq r\end{cases}
$$

is continuous and locally quasiconvex.

EXAMPLE 2. Let $M=\left(R^{2}, g\right)$ be a surface of revolution with finite area as in Figure 1. The exponential map at the point $p$ is injective on the tangent space $T_{p} M$. In particular, the single point set $\{p\}$ is totally convex. Hence, the function $\varphi: H \rightarrow R$ defined by $\varphi(p)=0, \varphi(q)=1(q \neq p)$ is quasiconvex. By the result of Yau [15], $M$ does not admit any nonconstant convex function.

The following example also indicates that there are a large number of Riemannian manifolds which admit locally nonconstant quasiconvex functions, but not locally nonconstant convex functions.

EXAMPLE 3 . Let $c: R \rightarrow R^{3}$ be a smooth curve parametrized by arc length such that $c$ is not a straight line and that the $\varepsilon$-tubular neighborhood $T_{\varepsilon}(c)$ of $c$ in $R^{3}$ is well defined for a positive number $\varepsilon$. We define $M$ to be the boundary of $T_{\varepsilon}(c)$. Let $e_{1}, e_{2}$ and $e_{3}$ be orthonormal frame fields along $c$ with $e_{1}(t)=c^{\prime}(t)$. M is parametrized by

$$
x(t, \theta)=c(t)+\varepsilon \cos \theta e_{2}(t)+\varepsilon \sin \theta e_{3}(t) .
$$

For every $t$, the curve $\gamma(\theta)=x(t, \theta)$ is a geodesic, so the image $\{x(t, \theta) ; \theta \in R\}$ must be totally convex. Since the set $\{x(t, \theta) ; t \leq a, \theta \in R\}$ is also totally convex for every $a \in R$, the function $\varphi: M \rightarrow R$ defined by $\varphi(x(t, \theta))=t$ is quasiconvex. By the argument in [9, Lemma 2], however, $M$ does not admit any locally nonconstant convex function. Otherwise, $M$ would split isometrically as a Riemannian product of the circle of radius $\varepsilon$ and the real line, and this would contradict the assumption on $c$. 


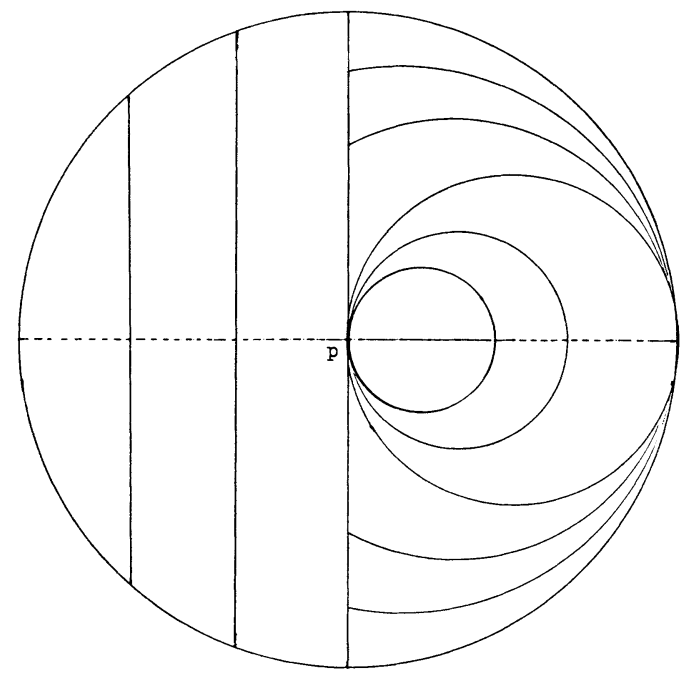

FIGURE 2

EXAMPLE 4. Let $f: R \rightarrow(-1,0)$ be a strictly increasing function. A locally nonconstant quasiconvex function $\varphi$ on the Euclidean plane $M=R^{2}$ is defined by

$$
\varphi(x, y)= \begin{cases}f(x) & \text { if } y \leq 0 \\ y & \text { if } y>0\end{cases}
$$

We note that $\partial M^{0}(\varphi)$ consists of the $x$-axis and that every distinct points $p$ and $q$ in $\partial M^{0}(\varphi)$ satisfy $\varphi(p) \neq \varphi(q)$.

EXAMPLE 5. Let $M$ be the Poincaré disk with constant negative curvature, and let $p$ be the center of $M$. Let $\gamma$ and $\sigma$ be unit speed geodesics in $M$ with $\gamma(0)=$ $\sigma(0)=p,\left\langle\gamma^{\prime}(0), \sigma^{\prime}(0)\right\rangle=0$. Let $f:(0, \infty) \rightarrow(-2,-1)$ and $g:[0, \infty) \rightarrow[-1,0)$ be strictly increasing functions. For every $t>0$, let $S_{t}$ denote the metric circle in $M$ of radius $t$ around $\gamma(t): S_{t}=\{q \in M ; d(\gamma(t), q)=t\}$. For every $t \leq 0$, let $H_{t}$ be the horocircle at $\gamma(\infty)$ through $\gamma(t)$, that is, $H_{t}$ is the boundary of the convex set $\bigcup_{s>t} B(\gamma(s), s-t)$. For every $t<0$, let $C_{t}$ be the Euclidean segment in $M$ perpendicular to $\gamma$ at $\gamma(t)$. The function $\varphi: M \rightarrow R$ defined by

$$
\varphi(x)= \begin{cases}-2 & \text { if } x=p \\ f(t) & \text { if } x \in S_{t}-\{p\} \\ g(0) & \text { if } x \in H_{0}-\{p\} \\ g(-t) & \text { if } x \in H_{t} \cap \bar{D} \\ -t & \text { if } x \in C_{t}\end{cases}
$$

is strictly quasiconvex with minimum -2 , where $D$ is the connected component of $M-\sigma((-\infty, \infty))$ containing $\gamma(1)$ (see Figure 2). We observe that although $\varphi$ is strictly quasiconvex, $\partial M^{0}(\varphi)$ consists of the geodesic $\sigma$.

EXAMPLE 6 . Let $M$ be the Euclidean plane. We set $I=\{(x, 0) ; 0 \leq x \leq 1\}$, and define the function $\varphi: M \rightarrow R$ by

$$
\varphi(x, y)= \begin{cases}1 & \text { if }(x, y) \in I \\ y & \text { if }(x, y) \notin I \text { and } y \geq 0 \\ |y|+1 & \text { if } y<0\end{cases}
$$




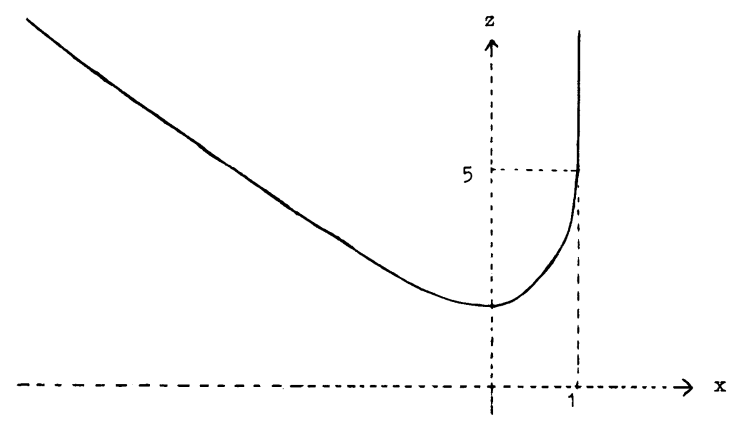

FIGURE 3

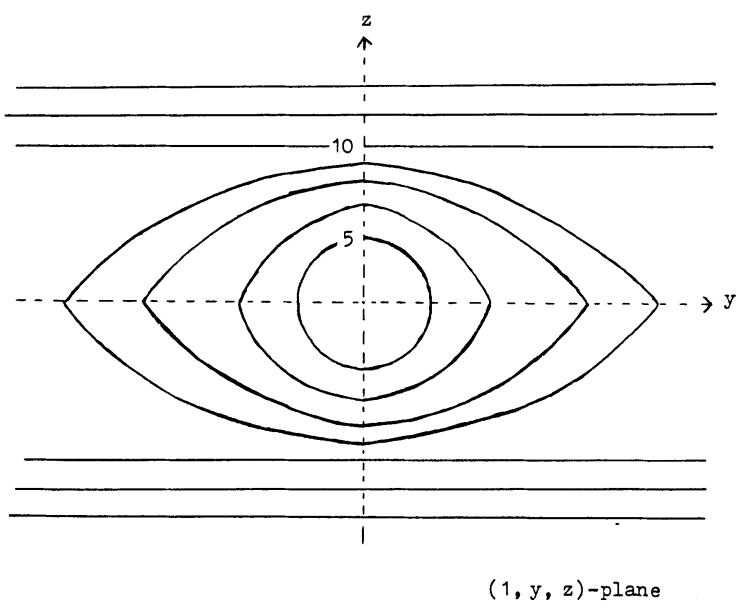

FIGURE 4

The function $\varphi$ is locally nonconstant and locally quasiconvex, but not quasiconvex.

EXAMPLE 7. For an arbitrary Riemannian manifold $N$, let $M=N \times R$ be the Riemannian product. For each quasiconvex function $f: R \rightarrow R \cup\{-\infty\}$, a quasiconvex function $\varphi$ on $M$ is defined by $\varphi(x, t)=f(t)$.

(1) Let $f(t)=t(t \geq 0), f(t)=-t+1(t<0)$. Then $M^{1}(\varphi)=N \times[0,1]$, and the minimum set $M^{0}(\varphi)$ is included in $\partial M^{1}(\varphi)$.

(2) Let $f(t)=t(t>0), f(t)=-t+1(t \leq 0)$. Then $\varphi$ has no minimum, but $M^{1}(\varphi)$ is bounded if $N$ is compact.

EXAMPLE 8. There is a complete metric on the cylinder $M=S^{1} \times R$ which admits a locally nonconstant continuous quasiconvex function $\varphi$ with minimum such that $\varphi^{-1}(a)$ is compact, but $\varphi^{-1}(c)$ is not compact for some $a>c>\inf _{M} \varphi$. Consider the "convex" curve in the $(x, z)$-plane described in Figure 3 which is tangent to the line $x=1$ at $z=5$. We define the function $\varphi$ on the surface of revolution of rotating the curve in $(x, y, z)$-space about the $x$-axis as follows: $\varphi(x, y, z)=|x|$ for $x<1$. For $x=1$, the level sets of $\varphi$ are described in Figure 4, and we define $\varphi$ there by $\varphi(1,0, z)=\psi(|z|)$, where $\psi:[0, \infty) \rightarrow[0, \infty)$ is a strictly 


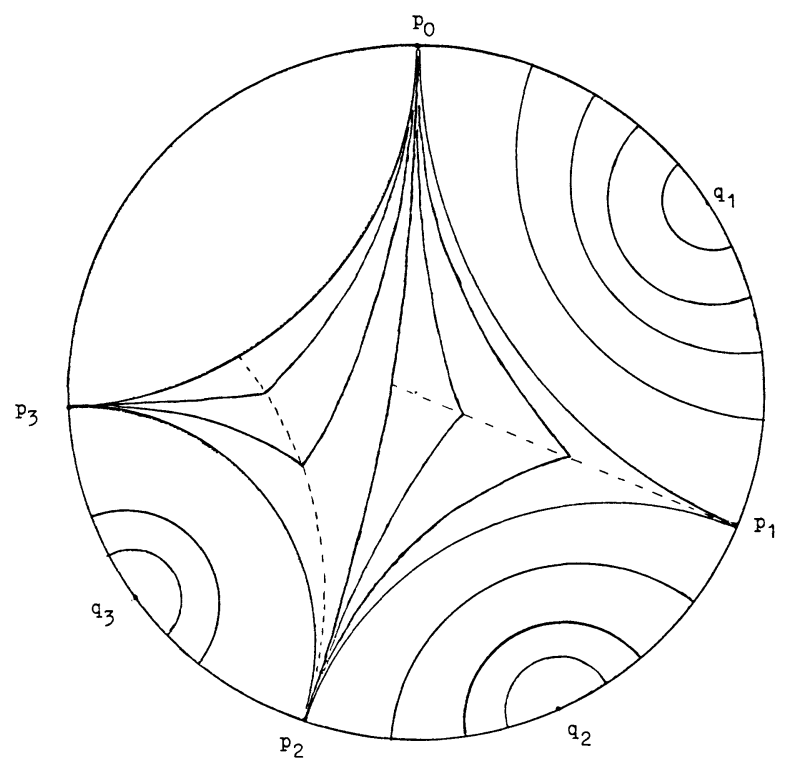

FIGURE 5

increasing continuous function with $\psi(5)=1, \psi(10)=10$ and

$$
\sup _{[0, \infty)}\{\psi(t) ; t \in[0, \infty)\}=20
$$

It is a locally nonconstant quasiconvex function with minimum 0 . Although $\varphi^{-1}(a)$ is compact for $a \geq 20, \varphi^{-1}(c)$ includes two lines for $10 \leq c<20$.

EXAMPLE 9. Let $M$ be the Poincaré disk and let $S^{1}=[0,1] /\{0,1\}$ be the boundary circle of $M$. We set $\bar{M}=M \cup S^{1}$. Take a sequence $0=p_{0}<p_{1}<p_{2}<\cdots$ of points in $S^{1}$ with $\lim p_{i}=1$. For each positive integer $i$, let $A_{2 i-1}\left(\right.$ resp. $A_{2 i}$ ) be the geodesic arc joining $p_{0}$ and $p_{i}$ (resp. joining $p_{i}$ and $p_{i+1}$ ). We define $\varphi \equiv-i$ on $A_{2 i-1} \cup A_{2 i}$. Now for each $i \geq 1$, take $q_{i} \in S^{1}$ with $p_{i-1}<q_{i}<p_{i}$ and choose continuous strictly monotone functions $f_{i}:[-i, \infty] \rightarrow S^{1}$ and $\dot{g}_{i}:[-i, \infty] \rightarrow S^{1}$ such that $f_{i}(-i)=p_{i}, g_{i}(-i)=p_{i+1}$ and $f_{i}(\infty)=g_{i}(\infty)=q_{i+1}$, where we assume that $f_{0}$ and $g_{0}$ are defined on $[-1, \infty]$ with $f_{0}(-1)=p_{0}, g_{0}(-1)=p_{1}$ and $f_{0}(\infty)=g_{0}(\infty)=q_{1}$. For every $i \geq 1$ and every $t \in[-i, \infty)$ (resp. every $t \in[-1, \infty)$ for $i=0)$, take a geodesic arc $B_{i, t}:[-\infty, \infty] \rightarrow \bar{M}$ such that $B_{i, t}(-\infty)=f_{i}(t)$ and $B_{i, t}(\infty)=g_{i}(t)$. We define $\varphi \equiv t$ on $B_{i, t}$. Next, for each $i \geq 1$, take an interior point $s_{i}$ of $A_{2 i+1}$. Let $\gamma_{i}:[-i-1,-i] \rightarrow \bar{M}$ be a geodesic (as image) with $\gamma_{i}(-i-1)=s_{i}, \gamma_{i}(-i)=p_{i}$. For each $s \in[-i-1,-i)$, let $C_{i, s}$ denote the union of the two geodesic arcs joining $p_{0}$ and $\gamma_{i}(s)$ and joining $\gamma_{i}(s)$ and $p_{i+1}$. We set $\varphi \equiv s$ on $C_{i, s}$. The function $\varphi$ on $M$ just constructed is a locally nonconstant continuous quasiconvex function such that $\partial M^{a}(\varphi)$ consists of infinitely many pairwise disjoint geodesic arcs (see Figure 5).

2. Locally convex sets and tangent cones. We recall some local properties of a locally convex set. See [6] for details. 
FACT $1[6]$. Let $A$ be a closed connected locally convex set in $M$. Then in the induced topology, $A$ carries the structure of imbedded submanifold of $M$ with (possibly empty) boundary $\partial A$. The interior Int $A=A-\partial A$ is a smooth totally geodesic submanifold of $M$. The boundary $\partial A$ need not be smooth nor connected.

If $A$ is a connected locally convex set, then the closure $\bar{A}$ of $A$ is also locally convex with Int $\bar{A} \subset A$. Hence we can define the boundary $\partial A$ and the interior Int $A$ of $A$ as $\partial A=\partial \bar{A}$ and $\operatorname{Int} A=\operatorname{Int} \bar{A}$. Now let $A$ be a locally convex set and let $\left\{A_{\alpha}\right\}_{\alpha}$ be the family of connected components of $A$. Since each component $A_{\alpha}$ is also locally convex, we can set Int $A=\bigcup_{\alpha} \operatorname{Int} A_{\alpha}, \partial A=\bigcup_{\alpha} \partial A_{\alpha}$.

FACT 2 [6]. For a closed locally convex set $A$ in $M$, if $\gamma:[0, \infty) \rightarrow M$ is a geodesic such that $\gamma([0,1)) \subset \operatorname{Int} A$ and $\gamma(1) \in \partial A$, then $\gamma(1+\varepsilon) \notin A$ for all sufficiently small $\varepsilon>0$.

Let $A$ be a closed locally convex set. The tangent cone $C_{p}(A)$ of $A$ at a point $p \in A$ is by definition the set

$$
\left\{v \in T_{p} M ; \exp _{p} \varepsilon v \in \operatorname{Int} A \text { for all sufficiently small } \varepsilon>0\right\},
$$

where $\exp _{p}: T_{p} M \rightarrow M$ is the exponential map. The tangent cone $C_{p}(A)$ is open in the subspace $\hat{C}_{p}(A)$ of $T_{p}(M)$ generated by $C_{p}(A)$.

FACT 3 [6]. The tangent cone $C_{p}(A)$ is a convex cone in $T_{p} M$.

PROOF. We give here a simple proof. For each $v_{1}, v_{2}$ in $C_{p}(A)$ and $\lambda \in[0,1]$, put $\gamma_{i}(t)=\exp _{p} t v_{i}(i=1,2), \gamma(t)=\exp _{p} t w$, where $w=(1-\lambda) v_{1}+\lambda v_{2}$. Note that $\gamma_{i}((0, \varepsilon]) \subset \operatorname{Int} A$ for an $\varepsilon>0$. For each $t \in(0, \varepsilon]$, let $\sigma_{t}$ be the minimizing geodesic segment from $\gamma_{1}(t)$ to $\gamma_{2}(t)$. Fact 2 shows $\sigma_{t}([0,1]) \subset$ Int $A$. Now an elementary calculation implies that the curve $\tau(t)=\sigma_{t}(\lambda)$ in Int $A$ is tangent to $w$, from which we have $w \in \overline{C_{p}(A)}$. This argument yields that $\overline{C_{p}(A)}$ is convex. Together with Fact 2, this also implies the convexity of $C_{p}(A)$.

If $p \in \partial A$, then $C_{p}(A)$ does not coincide with $\hat{C}_{p}(A)$ and is contained in an open half-space in $\hat{C}_{p}(A)$. Such an open half-space is called a supporting half-space for $A$ at $p \in \partial A$. A tangent vector $v$ to $M$ at $p \in A$ is called normal to $A$ at $p$ if $\langle v, w\rangle \leq 0$ for all $w \in C_{p}(A)$. The set $\nu_{p}(A)$ of all normal vectors to $A$ at $p$ is a closed convex cone in $T_{p}(M)$. If $p \in \partial A$, the intersection $\nu_{p}(A) \cap \hat{C}_{p}(A)$ coincides with the set of outward normals to the supporting half-spaces for $A$ at $p$. We consider the set $\nu(A)=\bigcup_{p \in A} \nu_{p}(A)$ with the induced topology from the tangent bundle $T M$ of $M$. We call $\nu(A)$ the normal bundle of $A$ in $M$. If $A$ has empty boundary, then $\nu(A)$ is the normal bundle in the usual sense.

We recall the following fact about a neighborhood of a closed locally convex set.

FACT $4[\mathbf{1 3}]$. If $A$ is a closed locally convex set in $M$, then there is an open neighborhood $U$ of $A$ in $M$ with the following properties:

(1) For each $q \in U$, there is a unique minimizing geodesic segment from $q$ to $A$, and this segment is entirely included in $U$.

(2) If $\pi(q)$ denotes the unique point of $A$ with $d(q, \pi(q))=d(q, A)$, then the mapping $\pi: U \rightarrow A$ is continuous. In fact, the restriction $\pi \mid(U-A)$ of $\pi$ to $U-A$ is of class $C^{1}$. The gradient vector of the distance function to $A$ at $q \in U-A$ is given by the unit vector tangent to the minimizing geodesic segment from $q$ to $\pi(q)$.

An open set $U$ with these properties is called a tubular neighborhood of $A$, and the mapping $\pi: U \rightarrow A$ is called the projection of $U$ onto $A$. The neighborhood $U$ is constructed as follows: Let $r: M \rightarrow(0, \infty)$ be a continuous function, called 
a convexity radius function, such that (i) for each $p \in M$ the ball $B(p, r(p)$ ) is convex, (ii) for every $q \in B(p, r(p))$ the function $x \rightarrow d^{2}(q, x)$ is strongly convex on $B(p, r(p))$, that is, its restriction to every nonconstant geodesic segment has positive second derivative. By definition, for each $p \in A$, there is $r_{1}(p) \in(0, r(p)]$ such that $A \cap B\left(p, r_{1}(p)\right)$ is convex. The neighborhood $U$ can be defined as $U=$ $\bigcup_{p \in A} B\left(p, r_{1}(p) / 2\right)$. It is straightforward to verify that $U$ satisfies properties (1) and (2) (cf. [7, Proposition 1.2]).

We note that $v \in \nu_{p}(A)$ if and only if $\pi\left(\exp _{p} \varepsilon v\right)=p$ for small $\varepsilon>0$. Thus $U$ is simply covered by geodesic segments normal to $A$. Hence we may take $U$ to be homeomorphic to $\nu(A)$.

The following proposition will be needed in $\S 5$ to construct a smooth vector field. For the proof, see [4, Lemma 11.1].

Proposition 2.1. If $C$ is an open convex cone in Euclidean space $R^{n}$ with $C \neq R^{n}$, then it contains an element $v$ such that $\Varangle(v, w)<\pi / 2$ for all $w \in C$, where $\Varangle(v, w)$ is the angle between the vectors $v$ and $w$.

3. Basic properties. From now on, $M$ will be assumed to be $n$-dimensional and complete. Let $\varphi: M \rightarrow R \cup\{-\infty\}$ be locally nonconstant and locally quasiconvex, unless otherwise stated. For a locally convex set $A$, we $\operatorname{denote}$ by $\operatorname{dim} A$ (resp. $\overline{\operatorname{dim}} A$ ) the smallest dimension (resp. the largest dimension) of connected components of $A$. Note that each sublevel set $M^{a}(\varphi)$ of $\varphi$ is locally convex.

LEMMA 3.1. The level set $\varphi^{-1}(a)$ is included in $\partial M^{a}(\varphi)$ for every $a \in \varphi(M)$ with $\underline{\operatorname{dim}} M^{a}(\varphi)=n$.

ProOF. The local quasiconvexity and local nonconstancy of $\varphi$ imply that $\varphi^{-1}([-\infty, a))$ is locally convex and is dense in $M^{a}(\varphi)$. Together with Fact 1 , this yields Int $M^{a}(\varphi) \subset \varphi^{-1}([-\infty, a))$ and $\varphi^{-1}(a) \subset \partial M^{a}(\varphi)$.

Since $\overline{M^{a}(\varphi)}$ is closed locally convex and $\overline{\operatorname{dim}} \overline{M^{a}(\varphi)}=\overline{\operatorname{dim}} M^{a}(\varphi)$ for every $a \in \varphi(M)$, it follows from the Baire Category Theorem that $\overline{\operatorname{dim}} M^{a}(\varphi)=n$ for some $a \in \varphi(M)$. We set

$$
\lambda_{1}=\inf \left\{a ; \overline{\operatorname{dim}} M^{a}(\varphi)=n\right\} \quad\left(\text { possibly, } M^{\lambda_{1}}(\varphi)=\varnothing\right) .
$$

LEMMA 3.2. If the set $M^{\lambda_{1}}(\varphi)$ is nonempty, then $\overline{\operatorname{dim}} M^{\lambda_{1}}(\varphi)<n$.

ProOF. Suppose $\overline{\operatorname{dim}} M^{\lambda_{1}}(\varphi)=n$. Let $N$ be a connected component of $M^{\lambda_{1}}(\varphi)$ with $\operatorname{dim} N=n$. The set $\varphi^{-1}\left(\left[-\infty, \lambda_{1}\right)\right) \cap \bar{N}$ is locally convex and is dense in $\bar{N}$. From the Baire Category Theorem, there would be some $a<\lambda_{1}$ with $\overline{\operatorname{dim}} M^{a}(\varphi) \cap$ $\bar{N}=n$, and this would contradict the definition of $\lambda_{1}$.

It should be noted that if $\varphi$ is continuous, locally nonconstant and locally quasiconvex, then it is quasiconvex. Otherwise, $\varphi \circ \gamma\left(t_{0}\right)=\sup \{\varphi \circ \gamma(t) ; 0 \leq t \leq 1\}>$ $\max \{\varphi \circ \gamma(0), \varphi \circ \gamma(1)\}$ for a geodesic $\gamma:[0,1] \rightarrow M$ and $t_{0} \in(0,1)$. If we set $a=\varphi \circ \gamma\left(t_{0}\right)$, it turns out $\gamma\left(t_{0}\right) \in \varphi^{-1}(a)=\partial M^{a}(\varphi)$ and $\gamma(0), \gamma(1) \in \operatorname{Int} M^{a}(\varphi)$. Since $\gamma([0,1]) \subset M^{a}(\varphi)$ and $M^{a}(\varphi)$ is locally convex, this contradicts Fact 2 . In the general case, we show that $\varphi$ is "almost quasiconvex" in the following sense (cf. Example 6).

LEMMA 3.3. For every $a>\lambda_{1}, M^{a}(\varphi)$ satisfies the following properties:

(1) $M^{a}(\varphi)$ is connected. 
(2) For all points $p \in \operatorname{Int} M^{a}(\varphi)$ and $q \in M^{a}(\varphi)$, Int $M^{a}(\varphi)$ includes all geodesic segments $\gamma:[0,1] \rightarrow M$ from $p$ to $q$ except for the end point $q$.

(3) Int $M^{a}(\varphi)$ is totally convex.

(4) For all points $x$ and $y$ in $\overline{M^{a}(\varphi)}$, there is a minimizing geodesic in $\overline{M^{a}(\varphi)}$ joining $x$ and $y$.

ProOF. We prove (1) and (2) at the same time. Take a connected component $U$ of $M^{a}(\varphi)$ with $\operatorname{dim} U=n$. For $p \in U \cap \operatorname{Int} M^{a}(\varphi)$ and $q \in M^{a}(\varphi)$, suppose that there is a geodesic segment $\gamma:[0,1] \rightarrow M$ from $p$ to $q$ such that $\gamma([0,1])$ is not included in $M^{a}(\varphi)$. The local quasiconvexity of $\varphi$ implies that there is a subdivision $0=s_{0}<s_{1}<\cdots<s_{k}=1$ such that $\varphi \circ \gamma(t) \leq \max \left\{\varphi \circ \gamma\left(s_{i}\right) ; 0 \leq i \leq k\right\}$ for all $t \in[0,1]$. Thus $\varphi$ has a maximum $b$ on $\gamma$. By the assumption and Lemma $3.1, \gamma((0,1))$ meets $\partial M^{b}(\varphi)$. Since $\gamma(0) \in \operatorname{Int} M^{b}(\varphi)$ and $\gamma([0,1]) \subset M^{b}(\varphi)$, this contradicts Fact 2 and shows (1). Together with Fact 2, this argument also shows that $\gamma([0,1])) \subset \operatorname{Int} M^{a}(\varphi)$ for all geodesics $\gamma$ from $p$ to $q$.

(3) This is clear from (2) and Fact 2.

(4) Take sequences $x_{i}$ and $y_{i}$ in Int $M^{a}(\varphi)$ with $x_{i} \rightarrow x$ and $y_{i} \rightarrow y$ as $i \rightarrow \infty$. Take minimizing geodesics $\gamma_{i}$ joining $x_{i}$ and $y_{i}$, which are included in Int $M^{a}(\varphi)$ by (3). The limit geodesic of a subsequence of $\gamma_{i}$ certainly lies in $\overline{M^{a}(\varphi)}$.

We now discuss a metric consequence of the existence of a locally quasiconvex function. The following proposition is closely related to the result of Yau [15] for a convex function.

PROPOSITION 3.4. Suppose that $M$ admits a locally quasiconvex function $\varphi$ satisfying one of the following:

(1) $\varphi$ is locally nonconstant.

(2) $\varphi$ is continuous, nonconstant and quasiconvex.

Then $M$ has infinite volume.

From Lemma 3.3, we know that for $a \in\left(\lambda_{1}, \sup _{M} \varphi\right)$, Int $M^{a}(\varphi)$ is a totally convex set of dimension $n$ with $\overline{M^{a}(\varphi)} \neq M$. Hence this is a direct consequence of the following

PROPOSITION 3.5. If $M$ has a proper totally convex set $A$ of dimension $n$, then $M$ has infinite volume.

Proof. Let $v$ be a unit element in the tangent cone $C_{p}(\bar{A})$ of $\bar{A}$ at a point $p$ of $\partial \bar{A}$. Take an $\varepsilon>0$ with $\exp \varepsilon v \in \operatorname{Int} A$ and $\exp -\varepsilon v \notin \bar{A}$. Suppose that $M$ has finite volume. By the Poincaré recurrence theorem, we can take unit vectors $v_{i}$ and positive numbers $t_{i}$ such that $v_{i} \rightarrow v, t_{i} \nearrow \infty$ and $F_{t_{i}}\left(v_{i}\right) \rightarrow v$ as $i \rightarrow \infty$, where $F_{t}$ is the geodesic flow on the unit tangent bundle. Let $U_{1} \subset \operatorname{Int} A$ and $U_{2} \subset M-\bar{A}$ be neighborhoods of $\exp \varepsilon v$ and $\exp -\varepsilon v$. For a sufficiently large $i, \exp \varepsilon v_{i} \in U_{1}$, $\exp \varepsilon F_{t_{i}}\left(v_{i}\right) \in U_{1}$ and $\exp -\varepsilon F_{t_{i}}\left(v_{i}\right) \in U_{2}$. If $\gamma$ is the geodesic with $\gamma^{\prime}(0)=F_{\varepsilon}\left(v_{i}\right)$, then $\gamma(0) \in A, \gamma\left(t_{i}-\varepsilon\right) \notin A$ and $\gamma\left(t_{i}+\varepsilon\right) \in A$. This contradicts the total convexity of $A$. Therefore $M$ has infinite volume.

REMARK 3.6. (1) When conditions (1) and (2) in Proposition 3.4 drop out, the proposition does not hold in general (see Examples 1 and 2).

(2) A slight modification of the proof of Proposition 3.5 shows that the proposition is still valid for a $n-1$ dimensional proper totally convex set $A$. 
LEMMA 3.7. Suppose that $\varphi$ is lower semicontinuous and $a \geq \lambda_{1}$. For every tubular neighborhood $U$ of $M^{a}(\varphi)$ and every compact set $K$ with $K \cap M^{a}(\varphi) \neq \varnothing$, there is $b \in \varphi(M), b>a$ with $M^{b}(\varphi) \cap K \subset U$.

This follows directly from the lower semicontinuity of $\varphi$.

LEMMA 3.8. Suppose that $\varphi$ is lower semicontinuous. Then for every two points in $M_{\varphi}=M^{\lambda_{1}}(\varphi)$, there is a minimizing geodesic segment joining them which is lying in $M_{\varphi}$. In particular, $M_{\varphi}$ is connected.

ProOF. For every point $x$ and $y$ in $M_{\varphi}$, let $B$ be a metric ball around $x$ containing $y$. By Lemma 3.7, for every positive $\varepsilon$, there is $c>\lambda_{1}$ such that $M^{c}(\varphi) \cap B \subset T_{\varepsilon}\left(M_{\varphi}\right)$. Lemma 3.3(4) shows that there is a minimizing geodesic segment $\gamma_{\varepsilon}$ in $M^{c}(\varphi)$ joining $x$ and $y$. Since $\gamma_{\varepsilon} \subset T_{\varepsilon}\left(M_{\varphi}\right)$, passing to a subsequence if necessary, we can conclude that $\gamma_{\varepsilon}$ converges to a geodesic in $M_{\varphi}$ as $\varepsilon \rightarrow 0$.

Proposition 3.9. Suppose that $\varphi$ is lower semicontinuous and that $M_{\varphi}$ is of dimension $n-1$ and has no boundary. Then $M_{\varphi}$ is totally convex.

Proof. Suppose that there is a geodesic segment $\sigma:[0,1] \rightarrow M$ such that $\sigma(0) \in M_{\varphi}$ and $\sigma(1) \in M_{\varphi}$ but $\sigma$ is not included in $M_{\varphi}$. By local quasiconvexity, $\varphi$ has a maximum $b>\lambda_{1}$ on $\sigma$. Since Int $\sigma$ meets $\partial M^{b}(\varphi)$, Fact 2 implies $\sigma \subset$ $\partial M^{b}(\varphi)$. In particular, $x:=\sigma(0) \in M_{\varphi} \cap \partial M^{b}(\varphi)$. We show $M_{\varphi} \subset \partial M^{b}(\varphi)$, which is a contradiction since $\sigma$ is transversal to $M_{\varphi}$ at $\sigma(0)$ and $\sigma \subset \partial M^{b}(\varphi)$. Let $v$ be a unit vector in $\nu_{x}\left(M^{b}(\varphi)\right)$. Since $\overline{C_{x}\left(M^{b}(\varphi)\right)} \supset C_{x}\left(M_{\varphi}\right)=T_{x} M_{\varphi}, v$ is unique and perpendicular to $T_{x} M_{\varphi}$. The tangent cone $C_{x}\left(M^{b}(\varphi)\right)$ coincides with the open half-space in $T_{x} M$ bounded by $T_{x} M_{\varphi}$ and with outward normal $v$. Using the fact that $M_{\varphi}$ is totally geodesic, we have $M_{\varphi} \subset \partial M^{b}(\varphi)$.

REMARK 3.10 . There is a $C^{1}$ surface $M$ which admits a lower semicontinuous locally nonconstant "locally quasiconvex" function $\varphi$ with not totally convex $M_{\varphi}$. In this case, a geodesic means a local isometry of $R$ into $M$. We take as $M$ a $C^{1}$-smoothly capped half-cylinder. More precisely, consider the curve $c$ in the $(x, z)$-plane defined by $x^{2}+z^{2}=1(z \leq 0)$ and $x= \pm 1(z \geq 0)$. Let $M$ be the surface of revolution of rotating the curve $c$ in the $(x, y, z)$-space about the $z$-axis. We set

$$
\begin{aligned}
S=\{(x, y, z) & \in M ; z \leq 0\}, \quad L=\{(x, 0, z) \in M ; z \leq 0\}, \\
p_{1} & =(-1,0,0), \quad p_{2}=(1,0,0) \in L .
\end{aligned}
$$

The set $S$ of the spherical part is covered with those minimizing geodesic segments $\gamma_{\theta}(0 \leq \theta \leq \pi / 2)$ joining $p_{1}$ and $p_{2}$ that make angle $\theta$ with $L$. We define $\varphi$ by

$$
\varphi(q)= \begin{cases}0 & \text { if } q \in L, \\ \theta & \text { if } q \in \partial_{\theta}-\left\{p_{1}, p_{2}\right\} \\ \pi / 2+z & \text { if } q=(x, y, z), z>0\end{cases}
$$

In the generalized sense, $\varphi$ is locally quasiconvex and $M_{\varphi}=L$ is not totally convex.

4. Reduction. For an arbitrary function $f: M \rightarrow R \cup\{-\infty\}$, we consider the function $f^{*}: M \rightarrow R \cup\{-\infty\}$ defined by

$$
f^{*}(p)=\inf \left\{a ; p \in \overline{M^{a}(f)}\right\} .
$$


The function $f^{*}$ is the lower limit function of $f$. We have immediately:

(1) $f^{*} \leq f$, that is, $M^{a}\left(f^{*}\right) \supset M^{a}(f)$ for all $a \in R \cup\{-\infty\}$.

(2) $\inf _{M} f^{*}=\inf _{M} f$. If $f$ has a minimum, then so does $f^{*}$.

(3) $f^{*}$ is lower semicontinuous, that is, $M^{a}\left(f^{*}\right)$ is closed for every $a \in R \cup\{-\infty\}$.

(4) $f$ is lower semicontinuous if and only if $f=f^{*}$.

Let $\varphi: M \rightarrow R \cup\{-\infty\}$ be locally quasiconvex.

LEMMA 4.1. $\varphi^{*}$ is also locally quasiconvex.

Proof. For every $x \in M$, take a convex ball $B$ around $x$ such that $\varphi \mid B$ is quasiconvex. We fix two points $p$ and $q$ in $B$. For every positive integer $i$, there are $p_{i}$ and $q_{i}$ such that $d\left(p, p_{i}\right)<1 / i, d\left(q, q_{i}\right)<1 / i, \varphi\left(p_{i}\right)<a+1 / i$ and $\varphi\left(q_{i}\right)<a+1 / i$, where $a:=\max \left\{\varphi^{*}(p), \varphi^{*}(q)\right\}$. If $\gamma_{i}$ is the minimizing geodesic segment joining $p_{i}$ and $q_{i}$, then the quasiconvexity of $\varphi \mid B$ shows $\gamma_{i} \subset M^{a+1 / i}(\varphi)$ for large $i$. Hence $M^{a}\left(\varphi^{*}\right)$ includes the minimizing geodesic segment joining $p$ and $q$, which is the limit of $\gamma_{i}$.

LEMMA 4.2. If $\varphi$ is locally nonconstant, then $\lambda_{1}=\inf \left\{a ; \overline{\operatorname{dim}} M^{a}\left(\varphi^{*}\right)=n\right\}$.

Proof. We set $\lambda_{1}^{*}=\inf \left\{a ; \overline{\operatorname{dim}} M^{a}\left(\varphi^{*}\right)=n\right\}$. Clearly $\lambda_{1}^{*} \leq \lambda_{1}$. Suppose $\lambda_{1}^{*}<\lambda_{1}$. Then $\overline{\operatorname{dim}} M^{\lambda_{1}-\varepsilon}\left(\varphi^{*}\right)=n$ for small positive $\varepsilon$. By the definition of $\varphi^{*}$, the set $\overline{M^{\lambda_{1}}(\varphi)}$ includes $M^{\lambda_{1}-\varepsilon}\left(\varphi^{*}\right)$. This contradicts Lemma 3.2

LEMMA 4.3. Suppose that $\varphi$ is locally nonconstant.

(1) If $a>\lambda_{1}$, then $M^{a}\left(\varphi^{*}\right)=\overline{M^{a}(\varphi)}$. In particular, $\partial M^{a}\left(\varphi^{*}\right)=\partial M^{a}(\varphi)$ and Int $M^{a}\left(\varphi^{*}\right)=\operatorname{Int} M^{a}(\varphi)$.

(2) $\varphi^{*}$ is also locally nonconstant.

PROOF. (1) To prove $M^{a}\left(\varphi^{*}\right)=\overline{M^{a}(\varphi)}$, it suffices to show $M^{a}\left(\varphi^{*}\right) \subset \overline{M^{a}(\varphi)}$. Suppose that there is a point $q$ in $M^{a}\left(\varphi^{*}\right)-\overline{M^{a}(\varphi)}$. From Lemma 3.3(4), for every $p \in \operatorname{Int} M^{a}(\varphi)$, there is a unit speed minimizing geodesic segment $\gamma:[0, d] \rightarrow M$ in $M^{a}\left(\varphi^{*}\right)$ from $q$ to $p$. Hence, we may assume $d$ is so small that $q$ and $p$ lie in a neighborhood $U$ on which $\varphi$ is quasiconvex. For each positive integer $i$, we may take $q_{i}$ with $d\left(q, q_{i}\right)<1 / i, \varphi\left(q_{i}\right)<a+1 / i$. For small $\varepsilon>0$, let $q^{\prime}=\gamma(\varepsilon)$ and let $\gamma_{i}:[0, d] \rightarrow M$ be the unit speed minimizing geodesic segment from $q_{i}$ through $q^{\prime}$. We may assume $q^{\prime} \notin \overline{M^{a}(\varphi)}$. Then it turns out $\varphi\left(q^{\prime}\right) \leq \max \left\{\varphi\left(q_{i}\right), \varphi\left(\gamma_{i}(d)\right)\right\}$, which is a contradiction since $\varphi\left(q_{i}\right)<a+1 / i<\varphi\left(q^{\prime}\right)$ and $\gamma_{i}(d) \in \operatorname{Int} M^{a}(\varphi)$ for large $i$.

(2) Suppose that there is an open set $U$ on which $\varphi$ takes the value $a$. We assert $a>\lambda_{1}$. By Lemma 3.2, we can take $p \in U$ such that $b:=\varphi(p)>\lambda_{1}$. Since $p \in \partial M^{b}(\varphi)$, we see $U-\overline{M^{b}(\varphi)} \neq \varnothing$. Hence $a+\varepsilon>b$ for every $\varepsilon>0$, because $x \in \overline{M^{a+\varepsilon}(\varphi)}$ for all $x \in U$. Thus $a \geq b>\lambda_{1}$. From (1) and Lemma 3.1, we have

$$
\begin{aligned}
\left(\varphi^{*}\right)^{-1}(a) & =M^{a}\left(\varphi^{*}\right)-\left(\varphi^{*}\right)^{-1}([-\infty, a)) \\
& \subset \overline{M^{a}(\varphi)}-\varphi^{-1}([-\infty, a)) \\
& \subset \overline{M^{a}(\varphi)}-\operatorname{Int} M^{a}(\varphi)=\partial M^{a}(\varphi) .
\end{aligned}
$$

This is a contradiction.

REMARK 4.4. As we see in the following, $M_{\varphi}=M^{\lambda_{1}}\left(\varphi^{*}\right)$ does not coincide with $\overline{M^{\lambda_{1}}(\varphi)}$ in general. 
(1) Let $M=N \times R$ be the Riemannian product. For the function $\varphi: M \rightarrow R$ given in Example 7(2), we observe $\lambda_{1}=0$ and $M^{0}(\varphi)=\varnothing$, but $M_{\varphi}=N \times 0$.

(2) Consider the function $\varphi: M \rightarrow R$ given in Example 6. We observe that $\lambda_{1}=0$ and $\overline{M^{0}(\varphi)}=\{(x, 0) ; x \leq 0$, or $x \geq 1\}$, but $M_{\varphi}$ coincides with the $x$-axis.

5. Construction of a generalized gradient vector field. Let $\varphi: M \rightarrow$ $R \cup\{-\infty\}$ be a locally nonconstant locally quasiconvex function.

LEMMA 5.1. For every $a>\lambda_{1}$, we have

$$
\operatorname{Int} M^{a}(\varphi)=\bigcup_{\lambda_{1}<c<a} \operatorname{Int} M^{c}(\varphi) .
$$

Proof. Clearly, Int $M^{c}(\varphi)$ is included in Int $M^{a}(\varphi)$ for every $c, \lambda_{1}<c<a$. Consider the union $A=\bigcup_{\lambda_{1}<c<a} M^{c}(\varphi)$, which is locally convex by the local quasiconvexity of $\varphi$. Since $\varphi$ is locally nonconstant, we have $\bar{A}=\overline{M^{a}(\varphi)}$, and hence Int $A=\operatorname{Int} M^{a}(\varphi)$. Hence it suffices to show Int $A=\bigcup_{\lambda_{1}<c<a} \operatorname{Int} M^{c}(\varphi)$. Clearly, Int $A \supset \bigcup_{\lambda_{1}<c<a}$ Int $M^{c}(\varphi)$. For every $x \in \operatorname{Int} A$, let $B$ be a convex ball around $x$ such that $\varphi \mid B$ is quasiconvex and $\bar{B} \subset \operatorname{Int} A$. We can take $n+1$ points $y_{0}, \ldots, y_{n}$ in $B$ such that the smallest closed convex set $C\left(y_{0}, \ldots, y_{n}\right)$ including the set $y_{0}, \ldots, y_{n}$ has dimension $n$ and contains $x$ as an interior point. Note that $B \cap M^{\alpha}(\varphi)$ is convex if it is nonempty. Since $\left\{y_{0}, \ldots, y_{n}\right\} \subset B \cap M^{c}(\varphi)$ for some $c \in\left(\lambda_{1}, a\right)$, we have

$$
C\left(y_{\mathrm{c}}, \ldots, y_{n}\right) \subset \overline{B \cap M^{c}(\varphi)} \subset \overline{M^{c}(\varphi)}
$$

Hence $x \in \operatorname{Int} M^{c}(\varphi)$.

We recall the definition of critical points of the distance function from a point in a complete manifold $M$ (cf. [12]). Let $p$ be a point in $M$ and let dist $p: M \rightarrow R$ be the distance function from $p$ : $\operatorname{dist}_{p}(x)=d(p, x)$. A point $q(\neq p)$ in $M$ is called a critical point of $\operatorname{dist}_{p}$ if the set $\mathcal{V}(q ; p)$ of all unit initial vectors to minimizing geodesic segments from $q$ to $p$ is not included in any open half-space of $T_{q} M$. For a subset $S$ of $M$, we also say that $q$ is a critical point of $\operatorname{dist}_{S}$ if the set $\mathcal{V}(q ; S):=\bigcup_{p \in S} \mathcal{V}(q ; p)$ is not included in any open half-space of $T_{q} M$.

LEMMA 5.2. For a compact set $K$ in $M$, let $q$ not be a critical point of $\operatorname{dist}_{K}$, and let $v$ be a vector in $T_{q} M$ such that $\Varangle(v, w)<\pi / 2$ for all $w \in \mathcal{V}(q ; K)$. Let $V$ be a smooth extension of $v$ to a neighborhood $U_{0}$ of $q$. Then, there is a neighborhood $U$ of $q$ such that $\Varangle(V(x), w)<\pi / 2$ for every $x \in U$ and every $w \in \mathcal{V}(x ; K)$.

PROOF. Suppose the contrary. Then there is a sequence $q_{i}$ in $U_{0}$ converging to $q$ such that $\Varangle\left(V\left(q_{i}\right), w_{i}\right) \geq \pi / 2$ for some $w_{i}$ in $\mathcal{V}\left(q_{i} ; K\right)$. Let $w$ be accumulation point of $w_{i}$ in the unit sphere bundle. By compactness and continuity, we have $w \in \mathcal{V}(q ; K)$ and $\Varangle(V(q), w) \geq \pi / 2$. This is a contradiction.

We denote by $C_{x}(\varphi)$ the tangent cone of $M^{\varphi(x)}(\varphi)$ at a point $x$ in $M$.

LEMMA 5.3. Suppose that $\varphi$ is lower semicontinuous. If $a>\lambda_{1}$, then each

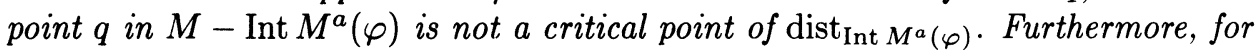
a compact set $K$ in Int $M^{a}(\varphi)$, there is some neighborhood $U$ of $q$ and a smooth vector field $V$ on $U$ satisfying the following conditions:

(1) $\Varangle(V(x), w)<\pi / 2$ for every $x$ in $U$ and every $w$ in $\mathcal{V}(x ; K)$. 
(2) (i) If $\varphi(q) \geq a$, then $V(x) \in C_{x}(\varphi)$ for all $x$ in $U$.

(ii) If $q \in \partial M^{a}(\varphi)$ and $\varphi(q)<a$, then $V(x) \in C_{x}(\varphi)$ for every $x$ in $U$ with $\varphi(x) \geq a$ and $V(x) \in C_{x}\left(M^{a}(\varphi)\right)$ for all $x$ in $\partial M^{a}(\varphi) \cap U$.

It should be noted that in the case $q \in \partial M^{a}(\varphi)$ and $\varphi(q)<a$, there is no assurance that $K \subset \operatorname{Int} M^{\varphi(q)}(\varphi)$. See Example 4 .

PROOF. If $\varphi(q) \geq a$ (resp. $q \in \partial M^{a}(\varphi)$ ), we take a vector $v$ in $C_{q}(\varphi)$ (resp. in $\left.C_{q}\left(M^{a}(\varphi)\right)\right)$ such that $\Varangle(v, w)<\pi / 2$ for all $w \in C_{q}(\varphi)$ (resp. for all $w \in$ $\left.C_{q}\left(M^{a}(\varphi)\right)\right)$. This is possible by Proposition 2.1 and Lemma 3.1. Lemma 3.3(2) implies $C_{q}(\varphi) \supset \mathcal{V}\left(q ;\right.$ Int $\left.M^{a}(\varphi)\right)$ (resp. $\left.C_{q}\left(M^{a}(\varphi)\right) \supset \mathcal{V}\left(q ; \operatorname{Int} M^{a}(\varphi)\right)\right)$. Hence, $q$ is not a critical point of $\operatorname{dist}_{\operatorname{Int} M^{a}(\varphi)}$, because $\Varangle(v, w)<\pi / 2$ for all $w$ in $\mathcal{V}\left(q ; \operatorname{Int} M^{a}(\varphi)\right)$. Let $V$ be a smooth extension of $v$ to a neighborhood $U$ of $q$. If $U$ is taken sufficiently small, then condition (1) is satisfied by Lemma 5.2. Moreover, the last condition (2)(ii) is satisfied as follows: Since $v \in C_{q}\left(M^{a}(\varphi)\right.$ ), we have $\exp \varepsilon V(q) \in \operatorname{Int} M^{a}(\varphi)$ for small $\varepsilon>0$. By continuity, $\exp \varepsilon V(x) \in \operatorname{Int} M^{a}(\varphi)$ for all $x$ sufficiently close to $q$. Hence $V(x)$ satisfies condition (2)(ii).

To assert that condition (2)(i) is satisfied, we suppose the contrary and derive a contradiction. Suppose that $\varphi(q) \geq a$ and that there is a sequence $q_{i}$ in $U$ converging to $q$ with $V\left(q_{i}\right) \notin C_{q_{i}}(\varphi)$. This can be divided into the following two cases. Let $\gamma$ and $\gamma_{i}$ be the geodesics with $\gamma^{\prime}(0)=V(q), \gamma_{i}^{\prime}(0)=V\left(q_{i}\right)$. Set $b=\varphi(q)$, $b_{i}=\varphi\left(q_{i}\right)$ and take $\varepsilon>0$ with $\gamma(\varepsilon) \in \operatorname{Int} M^{b}(\varphi)$.

Case (I). $b_{i} \geq b$ for infinitely many $i$.

Passing to a subsequence if necessary, we may assume $b_{i} \geq b$ for all $i$. Since $V\left(q_{i}\right) \notin C_{q_{i}}(\varphi)$, Lemma 3.3(2) shows that the $\gamma_{i}(t)$ are not contained in Int $M^{b_{i}}(\varphi)$ for all $t \geq 0$. Since $b_{i} \geq b>\lambda_{1}$, we have $\gamma_{i}(\varepsilon) \in M-\operatorname{Int} M^{b_{i}}(\varphi) \subset M-\operatorname{Int} M^{b}(\varphi)$ and $\gamma(\varepsilon)=\lim \gamma_{i}(\varepsilon) \in M-\operatorname{Int} M^{b}(\varphi)$. This is a contradiction.

Case (II). $b_{i}<b$ for infinitely many $i$.

We may assume $b_{i}<b$ for all $i$ as before. Since $\varphi$ is lower semicontinuous, $\varphi \circ \gamma(\delta) \rightarrow b$ as $\delta \rightarrow 0$. It follows from Lemma 5.1 that there is $c \in \varphi \circ \gamma((0, \varepsilon))$ such that $\gamma(\varepsilon) \in \operatorname{Int} M^{c}(\varphi)$ and $c>\lambda_{1}$. Since $c=\varphi(\gamma(t))<b$ for some $t \in$ $(0, \varepsilon), q=\gamma(0)$ is not contained in $M^{c}(\varphi)$. Thus it turns out that for large $i$, $q_{i} \notin M^{c}(\varphi)$ and $\gamma_{i}(\varepsilon) \in \operatorname{Int} M^{c}(\varphi) \in \operatorname{Int} M^{b_{i}}(\varphi)$ since $b_{i} \rightarrow b$. This contradicts the assumption $V\left(q_{i}\right) \notin C_{q_{i}}(\varphi)$.

The local vector fields constructed in the previous lemma patch together to form a global one with properties (1) and (2) in the lemma.

Proposition 5.4. Suppose that $\varphi$ is a lower semicontinuous. If $a>\lambda_{1}$ and $K$ is a compact subset of Int $M^{a}(\varphi)$, then there is a smooth complete vector field $X$ over $M$ with the following properties:

(1) $X$ has unit norm on $M-\operatorname{Int} M^{a}(\varphi)$.

(2) $\Varangle(X(q), w)<\pi / 2$ for every $q \in M-$ Int $M^{a}(\varphi)$ and every $w \in \mathcal{V}(q ; K)$.

(3) $X(q) \in C_{q}(\varphi)$ for every $q$ with $\varphi(q) \geq a$ and $X(q) \in C_{q}\left(M^{a}(\varphi)\right)$ for every $q \in \partial M^{a}(\varphi)$.

We call a vector field $X$ with the above properties a generalized gradient vector field of $\left(\varphi, \operatorname{dist}_{K}\right)$ supported on $M-\operatorname{Int} M^{a}(\varphi)$.

PROOF. Applying the previous lemma to each point in $M-\operatorname{Int} M^{a}(\varphi)$, we obtain a locally finite open covering $\left\{U_{i}\right\}_{i}$ of $M-\operatorname{Int} M^{a}(\varphi)$ and smooth vector fields $V_{i}$ on $U_{i}$ which satisfy the conditions in the lemma. Let $\left\{\rho_{i}\right\}_{i}$ be a partition of 
unity dominated with $\left\{U_{i}\right\}_{i}$. Then $X:=\sum \rho_{i} V_{i} /\left|\sum \rho_{i} V_{i}\right|$ makes sense as a smooth complete vector field over $M$. It is clear from the convexity of tangent cones and the construction of $X$ that $X$ has properties (1), (2) and (3).

Proposition 5.5. Let $X$ be a generalized gradient vector field of ( $\varphi$, $\operatorname{dist}_{K}$ ) supported on $M-\operatorname{int} M^{a}(\varphi)$. Each integral curve $\phi(t)$ of $X$ has the following properties:

(i) If $\varphi(\phi(0)) \geq a$, then $\varphi(\phi(t))$ is strictly decreasing as long as $\varphi(\phi(t)) \geq a$.

(ii) If $\phi(0) \in M$ - Int $M^{a}(\varphi)$, then $\operatorname{dist}_{p}(\phi(t))$ is strictly decreasing for every $p \in K$ as long as $\phi(t) \in M-\operatorname{Int} M^{a}(\varphi)$. The flow $\phi(t)$ goes to infinity as $t \rightarrow-\infty$ and intersects $\partial M^{a}(\varphi)$ in a single point.

As a result, we see that $M^{a}(\varphi)$ is a strong deformation retract of $M$.

PrOOF. (i) This is an immediate consequence of Lemma 3.1 and property (3) of $X$.

(ii) For fixed $p \in K$, let $B$ be a large metric ball around $p$ containing $\phi(0)$. From the compactness argument using the continuity of dist $_{p}$, it is easily checked that the number

$$
\delta=\inf \left\{\pi / 2-\Varangle(X(q), w) ; q \in B \cap\left(M-\operatorname{Int} M^{a}(\varphi)\right), w \in \mathcal{V}(q ; p)\right\}
$$

is strictly positive. The function $t \rightarrow \operatorname{dist}_{p}(\phi(t))$ is locally Lipschitz continuous, so differentiable almost everywhere. Since $X$ has unit norm on $M-\operatorname{Int} M^{a}(\varphi)$, the first variation formula implies that $(d / d t) \operatorname{dist}_{p}(\phi(t)) \leq-\sin \delta$ for almost all $t$ with $\phi(t) \in B \cap\left(M-\operatorname{Int} M^{a}(\varphi)\right)$, and hence that

$$
\begin{aligned}
\operatorname{dist}_{p}(\phi(t)) & =\operatorname{dist}_{p}(\phi(0))+\int_{0}^{t} \frac{d}{d t} \operatorname{dist}_{p}(\phi(t)) d t \\
& \leq \operatorname{dist}_{p}(\phi(0))-t \sin \delta
\end{aligned}
$$

for $t>0$, and $\operatorname{dist}_{p}(\phi(t)) \geq \operatorname{dist}_{p}(\phi(0))-t \sin \delta$ for $t<0$. This argument shows that the function $\operatorname{dist}_{p}(\phi(t))$ is strictly decreasing whenever $\phi(t) \in M-\operatorname{Int} M^{a}(\varphi)$, and that $\phi(t)$ goes to infinity as $t \rightarrow-\infty$. By property $(3)$ of $X$, the curve $\phi(t)$ intersects $\partial M^{a}(\varphi)$ in at most one point. Now suppose that $\phi(t)$ does not intersect $\partial M^{a}(\varphi)$. The curve $\phi(t), t \geq 0$, would have to always be lying in $B \cap\left(M-\operatorname{Int} M^{a}(\varphi)\right)$ because of $a>\lambda_{1}$. Put $d_{0}=\inf \left\{\operatorname{dist}_{p}(x) ; x \in \partial M^{a}(\varphi)\right\}$. The above calculation implies $\operatorname{dist}_{p}(\phi(t))<d_{0}$ for all $t>\left(\operatorname{dist}_{p}(\phi(0))-d_{0}\right) / \sin \delta$. Since this is a contradiction, the curve $\phi(t)$ intersects $\partial M^{a}(\varphi)$ in a single point.

COROLLARY 5.6. If $\varphi: M \rightarrow R \cup\{-\infty\}$ is a locally nonconstant locally quasiconvex function, then for every $a>\lambda_{1}, M-\operatorname{Int} M^{a}(\varphi)$ is homeomorphic to $\partial M^{a}(\varphi) \times[0, \infty)$ in such a way that in the case $\partial M^{a}(\varphi)$ is smooth, the homeomorphism becomes a diffeomorphism automatically.

ProOF. Proposition 5.5 is applicable to the function $\varphi^{*}$ by the argument in $\S 4$. We note that Lemma $4.3(1)$ shows $M^{a}\left(\varphi^{*}\right)=\overline{M^{a}(\varphi)}$. Take a point $p$ in Int $M^{a}(\varphi)$. Let $X$ be a generalized gradient vector field of $\left(\varphi^{*}\right.$, dist $\left._{p}\right)$ supported on $M$ - Int $M^{a}(\varphi)$. For each $q \in \partial M^{a}(\varphi)$, let $\phi_{q}(t)$ be the integral curve of $X$ with $\phi_{q}(0)=q$. Proposition 5.5 shows that the homeomorphism $F: \partial M^{a}(\varphi) \times[0, \infty) \rightarrow$ $M-$ Int $M^{a}(\varphi)$ can be defined by $F(q, t)=\phi_{q}(-t)$, and that if $\partial M^{a}(\varphi)$ is smooth, $F$ is actually a diffeomorphism. 
6. Proof of Theorem A. We begin with the reconstruction of a generalized gradient vector field with an additional condition.

LEMMA 6.1. Suppose that $\varphi: M \rightarrow R \cup\{-\infty\}$ is lower semicontinuous. Let $c>\lambda_{1}$ and let a compact set $K$ in $\operatorname{Int} M^{c}(\varphi)$ be given. Let $U_{0}$ be open and $H$ closed in $M$ with $H \subset U_{0}-M^{c}(\varphi)$. Let $T$ be a continuous vector field on $U_{0}-M^{c}(\varphi)$ with $T(x) \in \overline{C_{x}(\varphi)}$ for all $x \in U_{0}-M^{c}(\varphi)$. Then there is a generalized gradient vector field $X$ of $\left(\varphi\right.$, dist $\left._{K}\right)$ supported on $M$ - Int $M^{c}(\varphi)$ with $\Varangle(X(q), T(q))<\pi / 2$ for all $q \in H$.

Proof. For each $q \in U_{0}-M^{c}(\varphi)$, take a vector $v$ in $C_{q}(\varphi)$ such that $\Varangle(v, w)<$ $\pi / 2$ for all $w \in C_{q}(\varphi)$. The set $\mathcal{V}(q ; K)$ is compact in the open convex cone $C_{q}(\varphi)$. Hence, we can take $\hat{v} \in C_{q}(\varphi)$ sufficiently close to $v$ so that $T(q)$ and $\mathcal{V}(q ; K)$ are included in the open half-space with outward normal $-\hat{v}$. Using the argument in Lemma 5.3, we extend $\hat{v}$ to a smooth vector field $V_{q}$ on a neighborhood $U_{q}$ of $q$ in $U_{0}-M^{c}(\varphi)$ so as to satisfy

(1) $\Varangle\left(V_{q}(x), T(x)\right)<\pi / 2, \Varangle\left(V_{q}(x), w\right)<\pi / 2$,

(2) $V_{q}(x) \in C_{x}(\varphi)$

for every $x \in U_{q}$ and every $w \in \mathcal{V}(q ; K)$. For $q$ in $\left(M-\operatorname{Int} M^{c}(\varphi)\right)-\left(U_{0}-M^{c}(\varphi)\right)$, take $U_{q}$ and $V_{q}$ in the same way as in Lemma 5.3, where we may assume $U_{q} \cap K=\varnothing$. The argument in Proposition 5.4 shows that these local vector fields patch together to form a required global one.

ProOF OF THEOREM A. We assume that $M_{\varphi}$ is noncompact, because the compact case is simpler. Let $U_{0}$ and $U$ be tubular neighborhoods of $M_{\varphi}$ with $\bar{U} \subset U_{0}$, and let a point $p$ in $M_{\varphi}$ be fixed. We may assume $d(x, \pi(x)) \leq 1 / 10$ for all $x \in U_{0}$, where $\pi: U \rightarrow M_{\varphi}$ is the projection. We set $\varepsilon=1 / 100, B_{i}=B(p, 10 i)$, $B_{i}^{\prime}=B(p, 10 i+5)(i=1,2, \ldots)$. Choose a sequence $\varepsilon_{1} \geq \varepsilon_{2} \geq \ldots$ with $\varepsilon \geq \varepsilon_{i}>0$ such that the $\varepsilon_{i}$-tubular neighborhoods $D_{i}=\left\{x ; \pi(x) \in M_{\varphi} \cap B_{i+1}, d(x, \pi(x))<\varepsilon_{i}\right\}$ of $M_{\varphi} \cap B_{i+1}$ are included in $U$. Since the function $x \mapsto d(x, \pi(x))$ is of class $C^{1}$ on $U-M_{\varphi}$, the boundaries $L_{i}$ of $D_{i}$ are of class $C^{1}$. We first construct a $C^{1}$ hypersurface $\hat{L}$ by connecting these $L_{i}$ as follows: We set $A_{i}=\left\{x \in L_{i} ; 10 i+2 \leq\right.$ $d(p, \pi(x)) \leq 10 i+3\} \subset B_{i}^{\prime}-B_{i}$. Let $f_{i}: A_{i} \rightarrow\left[\varepsilon_{i+1}, \varepsilon_{i}\right]$ be $C^{1}$ functions such that

$$
f_{i}= \begin{cases}\varepsilon_{i} & \text { on a neighborhood of }\left\{x \in A_{i} ; d(p, \pi(x))=10 i+2\right\}, \\ \varepsilon_{i+1} & \text { on a neighborhood of }\left\{x \in A_{i} ; d(p, \pi(x))=10 i+3\right\}\end{cases}
$$

For each $x \in A_{i}$, let $\gamma_{x}:\left[0, \varepsilon_{i}\right] \rightarrow M$ be the minimizing geodesic from $\pi(x)$ to $x$, and let $C_{i}=\left\{\gamma_{x}\left(f_{i}(x)\right) ; x \in A_{i}\right\}$. By replacing $A_{i}$ with $C_{i}$, we can connect $L_{i}$ and $L_{i+1}$ and construct the $C^{1}$ hypersurface $\hat{L}$. If $\partial M_{\varphi}=\varnothing$, we may assume that $\hat{L}$ is smooth. We also note that if $x \in \hat{L}$ and $\pi(x) \in B_{i}-B_{i-1}^{\prime}$, then $x \in L_{i}$.

Let $\hat{U}$ be the open neighborhood of $M_{\varphi}$ bounded by $\hat{L}$ and let $U_{1}$ be a tubular neighborhood of $M_{\varphi}$ with $\bar{U}_{1} \subset \hat{U}$. By Lemma 3.7, we can take $c_{i} \in \varphi^{*}(M)$ with $c_{i}>\lambda_{1}, c_{1} \geq c_{2} \geq \cdots$ so that $M^{c_{i}}\left(\varphi^{*}\right) \cap B_{i+1} \subset U_{1}$. It should be noted that there is no assurance that $M_{\varphi} \subset \operatorname{Int} M^{c_{i}}\left(\varphi^{*}\right)$. Let $T$ be the unit vector field on $U_{0}-M_{\varphi}$ such that $T(x)$ is tangent to the minimizing geodesic segment from $x$ to $M_{\varphi}$. Take $p_{i} \in \operatorname{Int} M^{c_{i+1}}\left(\varphi^{*}\right) \cap B(p, \varepsilon)$ and generalized gradient vector fields $X_{i}$ of $\left(\varphi^{*}, \operatorname{dist}_{\left\{p_{i-1}, p_{i}, p_{i+1}\right\}}\right)$ supported on $M-\operatorname{Int} M^{c_{i}}\left(\varphi^{*}\right)$ with $\left|X_{i}\right| \leq 1$ on $M$. From Lemma 6.1, we may assume $\Varangle\left(X_{i}(q), T(q)\right)<\pi / 2$ for all $q \in \bar{B}_{i+1} \cap\left(\bar{U}-U_{1}\right)$. Let 
$\rho_{i}: M \rightarrow R$ be smooth functions with $0 \leq \rho_{i} \leq 1$ such that

$$
\rho_{i}= \begin{cases}1 & \text { on } B_{i}-B_{i-1}, \\ 0 & \text { outside a small neighborhood of } \overline{\left(B_{i}-B_{i-1}\right)} .\end{cases}
$$

If $X:=\sum_{i=1}^{\infty} \rho_{i} X_{i}$, we may assume $|X| \leq 2$ over $M$. Let $\delta: \hat{L} \rightarrow R$ be a positive function of class $C^{1}$ such that for every $x \in \hat{L}$, the neighborhood $\hat{U}-\bar{U}_{1}$ contains the point $y(x)$ on the minimizing geodesic segment from $x$ to $\pi(x)$ with $d(x, y(x))=$ $\delta(x)$. Let $U_{\delta}$ be the open neighborhood of $M_{\varphi}$ bounded by the $C^{1}$ hypersurface $L_{\delta}=\{y(x) ; x \in \hat{L}\}$. We note $U_{1} \subset \bar{U}_{1} \subset U_{\delta} \subset \bar{U}_{\delta} \subset \hat{U}, \partial U_{\delta}=L_{\delta}$ and $\partial \hat{U}=\hat{L}$. Let $\rho, \mu: M \rightarrow R$ be smooth functions with $0 \leq \rho, \mu \leq 1$ such that

$$
\rho=\left\{\begin{array}{ll}
1 & \text { on } M-\hat{U}, \\
0 & \text { on } \bar{U}_{\delta},
\end{array} \quad \mu= \begin{cases}1 & \text { on } M-U_{\delta} \\
0 & \text { on } \bar{U}_{1} .\end{cases}\right.
$$

Then $Y:=\rho X+\mu(1-\rho) T$ makes sense as a smooth complete vector field over $M$.

ASSERTION. The function $\delta$ can be taken so small that for every $x \in M-U_{\delta}$, the integral curve $\phi_{x}(t)$ of $Y$ with $\phi_{x}(0)=x$ intersects $L_{\delta}$ transversally with a single point.

We assume this for the moment. The integral curves of $Y$ give rise to a $C^{1}$ diffeomorphism of $M-U_{\delta}$ onto $L_{\delta} \times[1, \infty)$, which fits with the $C^{1}$ diffeomorphism of $\bar{U}_{\delta}-M_{\varphi}$ onto $L_{\delta} \times(0,1]$ to form a $C^{1}$ diffeomorphism of $M-M_{\varphi}$ onto $L_{\delta} \times(0, \infty)$. It follows from a basic result in differential topology that $L_{\delta}$ contains a smooth structure and that the $C^{1}$ diffeomorphism between $M-M_{\varphi}$ and $L_{\delta} \times(0, \infty)$ can be approximated by a (smooth) diffeomorphism. For every $x \in L_{\delta}$, let $\sigma_{x}:[0, \varepsilon(x)] \rightarrow$ $M$ be the minimizing geodesic segment from $\pi(x)$ to $x$, where $\varepsilon(x)=d(x, \pi(x))$. Let $\psi_{x}:[0, \infty) \rightarrow M$ be the extension of $\sigma_{x}$ to $\sigma_{x} \cup \phi_{x}$, that is, $\psi_{x} \mid[0, \varepsilon(x)]=$ $\sigma_{x}$ and $\psi_{x}(t)=\phi_{x}(\varepsilon(x)-t)$ for $t \geq \varepsilon(x)$. Then the correspondence $\sigma_{x}(t) \mapsto$ $\psi_{x}(\tan (\pi t / 2 \varepsilon(x)))$ gives rise to a homeomorphism of $U_{\delta}$ onto $M$. Therefore $M$ is homeomorphic to the normal bundle $\nu\left(M_{\varphi}\right)$. If $\partial M_{\varphi}=\emptyset$, then $M$ is diffeomorphic to $U_{\delta}$, and to $\nu\left(M_{\varphi}\right)$.

PROOF OF ASSERTION. If we set $\kappa_{i}=\inf \left\{\langle T, X\rangle(x) ; x \in \bar{B}_{i} \cap\left(\bar{U}-U_{1}\right)\right\}>0$ and $\eta_{i}=\min \left\{1, \kappa_{i}\right\}$, then we see $\langle Y, T\rangle=\rho\langle X, T\rangle+(1-\rho)\langle T, T\rangle \geq \eta_{i}$ on $\bar{B}_{i} \cap\left(\bar{U}-U_{1}\right)$. Take $\delta$ so as to satisfy $\delta(x)<\eta_{i+1} / 2$ for all $x \in \hat{L} \cap\left(B_{i}-B_{i-1}\right)$. Since $Y$ is transversal to $L_{\delta}$, it suffices to show that for every $x \in B_{i}-U_{\delta}$, the integral curve $\phi_{x}(t)$ intersects the set $\left\{y \in L_{\delta} ; d(p, \pi(y)) \leq 10 i+2\right\}$. Since $x \in B\left(p_{i}, 10 i+\varepsilon\right)$ and $Y=X$ on $M-\hat{U}$, $\operatorname{dist}_{p_{i}}\left(\phi_{x}(t)\right)$ is strictly decreasing as long as $\phi_{x}(t) \in$ $B\left(p_{i}, 10 i+\varepsilon\right)-U$. Hence there is $t_{0} \geq 0$ such that $\phi_{x}\left(t_{0}\right) \in B\left(p_{i}, 10 i+\varepsilon\right) \cap\left(\hat{U}-U_{\delta}\right) \subset$ $B(p, 10 i+2 \delta) \cap\left(\hat{U}-U_{\delta}\right)$. We consider the following two cases.

Case (I). $\phi_{x}\left(t_{0}\right) \in\left(B(p, 10 j+2 \varepsilon)-B_{j-1}^{\prime}\right) \cap\left(\hat{U}-U_{\delta}\right)$ for some $j \leq i$.

We first note that $d\left(\phi_{x}\left(t_{0}\right), M_{\varphi}\right) \leq \varepsilon_{j}$ and that $\phi_{x}(t) \in B(p, 10 j+1+2 \varepsilon)-$ $B(p, 10 j-6)$ for every $t \in\left[t_{0}, t_{0}+1 / 2\right]$ since $|Y| \leq 2$. It follows

$$
\begin{aligned}
d\left(\phi_{x}(t), M_{\varphi}\right) & \left.=d\left(\phi_{x}\left(t_{0}\right), M_{\varphi}\right)+\int_{t_{0}}^{t} \frac{d}{d t} d\left(\phi_{x}(t), M_{\varphi}\right)\right) d t \\
& =d\left(\phi_{x}\left(t_{0}\right), M_{\varphi}\right)-\int_{t_{0}}^{t}\langle Y, T\rangle\left(\phi_{x}(t)\right) d t \\
& \leq \varepsilon_{j}-\kappa_{j+1}\left(t-t_{0}\right) \leq \varepsilon_{j}-\eta_{j+1}\left(t-t_{0}\right) .
\end{aligned}
$$


Thus we must have $\phi_{x}(t) \in L_{\delta}$ for some $t \in\left[t_{0}, t_{0}+1 / 2\right]$.

Case (II). $\phi_{x}\left(t_{0}\right) \in\left(B_{j}^{\prime}-B_{j}\right) \cap\left(\hat{U}-U_{\delta}\right)$ for some $j \leq i-1$.

Suppose that $\phi_{x}(t) \in B(p, 10 j+5+3 \varepsilon)-U_{\delta}$ for all $t \geq t_{0}$. Since

$$
\inf \left\{\langle Y, T\rangle\left(\phi_{x}(t)\right) ; t \geq t_{0}\right\} \geq \kappa_{j+1},
$$

it turns out $\phi_{x}(t) \in U_{\delta}$ for some $t<t_{0}+\varepsilon_{j} / \kappa_{j+1}$. This is a contradiction. Hence we may assume $\phi_{x}\left(t_{1}\right) \in B(p, 10 j+5+3 \varepsilon)$ for some $t_{1}>t_{0}$. Since $d\left(p_{j}, \phi_{x}\left(t_{0}\right)\right)<$ $10 j+5+\varepsilon$ and $d\left(p_{j}, \phi_{x}\left(t_{1}\right)\right)>10 j+5+2 \varepsilon$, there are $r \in[10 j+5+\varepsilon, 10 j+5+2 \varepsilon]$ and $t_{2} \in\left(t_{0}, t_{1}\right)$ such that $d\left(p_{j}, \phi_{x}\left(t_{2}\right)\right)=r$ and $d\left(p_{j}, \phi_{x}(t)\right)>r$ for every $t \in\left(t_{2}, t_{1}\right]$. Since the function $t \mapsto \operatorname{dist}_{p_{j}}\left(\phi_{x}(t)\right)$ is strictly decreasing as long as $\phi_{x}(t) \in M-\hat{U}$, we see $\phi_{x}\left(t_{2}\right) \in \hat{U}$. We note $\phi_{x}\left(t_{2}\right) \in\left(B_{j+1}-B_{j}^{\prime}\right) \cap \hat{U}$. Hence by Case $(\mathrm{I}), \phi_{x}(t)$ meets $L_{\delta}$.

7. Special cases. In this section, we consider the case where a locally nonconstant locally quasiconvex function $\varphi: M \rightarrow R \cup\{-\infty\}$ has a sublevel set of maximal dimension whose boundary includes a compact connected component, and prove Theorems B and C. For this, we need some lemmas. From now on, let $\varphi: M \rightarrow R \cup\{-\infty\}$ be a locally nonconstant locally quasiconvex function.

LEMMA 7.1. Suppose that for some $a>\lambda_{1}, \partial M^{a}(\varphi)$ has a compact connected component $L$. For some $c \in\left(\lambda_{1}, a\right]$, suppose that there is a continuous curve $\sigma:[0,1] \rightarrow M$ such that $\sigma(0) \in L, \sigma([0,1]) \cap \partial M^{c}(\varphi) \neq \varnothing$ and $\sigma([0,1]) \subset$ $\overline{M^{a}(\varphi)}-$ Int $M^{c}(\varphi)$. Then there is a compact set $K$ in $M$ satisfying the following conditions:

(1) $\sigma([0,1]) \subset K \subset \overline{M^{a}(\varphi)}-\operatorname{Int} M^{c}(\varphi)$.

(2) If $L_{1}$ denotes the connected component of $\partial M^{c}(\varphi)$ containing a point of $\sigma([0,1]) \cap \partial M^{c}(\varphi)$, then it is homeomorphic to $L$ and includes $\sigma([0,1]) \cap \partial M^{c}(\varphi)$. Moreover, $\partial K=L \cup L_{1}$, where $\partial K=K \cap \overline{(M-K)}$.

(3) If Int $K=\varnothing$, then $K=L$, where Int $K=K-\partial K$. In the case Int $K \neq \varnothing$, for each $y \in \operatorname{Int} K$, if $L_{y}$ denotes the connected component of $\partial M^{\varphi^{*}(y)}\left(\varphi^{*}\right)$ containing $y$, then it is homeomorphic to $L$ and included in $K$.

(4) $K$ is included in $B(p, R)$ for every $p \in \overline{M^{c}(\varphi)}$ and $R>0$ with $L \subset B(p, R)$.

(5) In the case $L \cap \overline{M^{c}(\varphi)}=\varnothing, K$ is homeomorphic to $L \times[0,1]$.

Proof. Let $p$ be any point in $\operatorname{Int} M^{c}(\varphi)=\operatorname{Int} M^{c}\left(\varphi^{*}\right)$, and let $X$ be a generalized gradient vector field of $\left(\varphi^{*}\right.$, dist $\left._{p}\right)$ supported on $M-\operatorname{Int} M^{c}\left(\varphi^{*}\right)$. For each $x$, let $\phi_{x}(t)$ be the integral curve of $X$ with $\phi_{x}(0)=x$. From Proposition 5.4, for every $x \in L$, there is unique $s(x) \geq 0$ such that $\phi_{x}(s(x)) \in \partial M^{c}\left(\varphi^{*}\right)$. We note that $X$ is transversal to $L$ at every point $x$ of $L$, because $X(x) \in C_{x}\left(M^{a}\left(\varphi^{*}\right)\right)$. Define the mapping $F: L \times[0,1] \rightarrow M$ by $F(x, t)=\phi_{x}(s(x) t)$ and put $K=F(L \times[0,1])$. We have to show that $K$ satisfies the given conditions.

(1) From the construction, certainly $K \subset M^{a}\left(\varphi^{*}\right)-\operatorname{Int} M^{c}\left(\varphi^{*}\right)$. Now consider the set

$$
I=\left\{s \in[0,1] ; \phi_{\sigma(s)}(t)(t \leq 0) \text { meets } L\right\} .
$$

The set $I$ is closed, because the mapping $(q, t) \rightarrow \phi_{q}(t)$ is continuous and $L$ is closed. Moreover $I$ is open, because $L$ is a compact hypersurface of $M$ without boundary. Since $0 \in I$, we have $I=[0,1]$ and $\sigma([0,1]) \subset K$. 
(2) The argument in (1) also shows $F(L \times\{1\}) \supset \sigma([0,1]) \cap \partial M^{c}\left(\varphi^{*}\right)$. Since the correspondence $x \mapsto \phi_{x}(s(x))$ is continuous and injective, $F(L \times\{1\})$ is a compact submanifold of $\partial M^{c}\left(\varphi^{*}\right)$ homeomorphic to $L$. Hence it must be a connected component of $\partial M^{c}\left(\varphi^{*}\right)$ and coincides with $L_{1}$. It remains to show $\partial K=L \cup L_{1}$. Clearly $L \cup L_{1} \subset \partial K$. We show $\partial K \subset L \cup L_{1}$. For $z \in \partial K$ with the representation $z=\phi_{x}(s(x) t), x \in L$, suppose $s(x) \neq 0$ and $t \neq 0,1$. Then there is a neighborhood $U$ of $x$ in $L$ such that $s\left(x^{\prime}\right) \neq 0$ for all $x^{\prime} \in U$. It turns out that $K$ includes a neighborhood of $z$ in $M$. This is a contradiction. Hence $z \in L \cup L_{1}$.

(3) If Int $K=\varnothing$, we observe that $s(x)=0$ for all $x \in L$, and $K=L$. Consider the case Int $K \neq \varnothing$. Since Int $K \subset \operatorname{Int} M^{a}\left(\varphi^{*}\right)-M^{c}\left(\varphi^{*}\right)$, we see $\varphi^{*}(y) \in(c, a)$ for each $y \in \operatorname{Int} K$. We may consider $X$ as a generalized gradient vector field of $\left(\varphi^{*}\right.$, dist $\left._{p}\right)$ supported on $M-\operatorname{Int} M^{b}\left(\varphi^{*}\right), b:=\varphi^{*}(y)$. Proposition 5.5 shows that for every $x \in L$, there is unique $\tilde{s}(x) \in[0, s(x)]$ with $\phi_{x}(\tilde{s}(x)) \in \partial M^{b}\left(\varphi^{*}\right)$. Similarly to (2), we have $L_{y}=\left\{\phi_{x}(\tilde{s}(x)) ; x \in L\right\}$.

(4) The set $K$ is uniquely determined by given $L$ and $c$ and independent of $p$ as is checked by the following: For any other point $p^{\prime}$ in $\operatorname{Int} M^{c}\left(\varphi^{*}\right)$, construct $K^{\prime}$ in the same manner. Applying (1) to each curve $t \mapsto F(x, t)(0 \leq t \leq 1)$, we have $F(x, t) \in K^{\prime}$ and $K=K^{\prime}$. The decreasing property of the function $\operatorname{dist}_{p}\left(\phi_{x}(t)\right)$ shows $K \subset B(p, R)$ for every $p \in \operatorname{Int} M^{c}\left(\varphi^{*}\right)$ and $R>0$ with $L \subset B(p, R)$. Therefore, the assertion is valid.

(5) This follows from the fact that $s(x)>0$ for all $x \in L$.

LEMMA 7.2. Suppose that for some a $>\lambda_{1}, \partial M^{a}(\varphi)$ has a compact connected component $L$. If $M_{\varphi}$ is nonempty, it is compact.

ProOF. Suppose that $M_{\varphi}$ is not compact. Then it is unbounded since $\varphi^{*}$ is lower semicontinuous. We note that $L$ is not included in $M_{\varphi}$. Otherwise, $L$ is a connected component of $M_{\varphi}$, because $\operatorname{dim} M_{\varphi}<n$. Since $M_{\varphi}$ is connected, it turns out $L=M_{\varphi}$. This is impossible by the assumption. We first carry $L$ near $M_{\varphi}$ using Lemma 7.1. For $q \in L-M_{\varphi}$, take $x_{0} \in M_{\varphi}$ and a unit speed minimizing geodesic $\sigma_{0}:\left[0, d_{0}\right] \rightarrow M$ from $x_{0}$ to $q$ with $d\left(q, x_{0}\right)=d\left(q, M_{\varphi}\right)$ and $\sigma_{0}\left(\left[0, d_{0}\right]\right) \subset M^{a}\left(\varphi^{*}\right)$. For $R>0$ with $L \subset B\left(x_{0}, R\right)$, put

$$
\begin{gathered}
\delta=\frac{1}{2} \inf \left\{r(x) ; x \in B\left(x_{0}, 2 R\right)\right\}, \quad \varepsilon=\delta / 10, \quad \hat{\varepsilon}=\min \left\{\varepsilon, d_{0}\right\}, \\
c_{0}=\inf \left\{\varphi^{*}\left(\sigma_{0}(t)\right) ; \hat{\varepsilon} \leq t \leq d_{0}\right\}>\lambda_{1},
\end{gathered}
$$

where $r$ is a convexity radius function over $M$. Lemma 7.1 shows that the point $q_{0}^{\prime}:=\sigma_{0}(\hat{\varepsilon})$ is contained in a compact set $L_{0}$ in $B\left(x_{0}, R\right)$ homeomorphic to $L$. Take a unit speed minimizing geodesic ray $\gamma:[0, \infty) \rightarrow M$ emanating from $x_{0}$ with $\gamma([0, \infty)) \subset M_{\varphi}$. By our assumption and Lemma 3.8, such $\gamma$ certainly exists. Put $x_{1}=\gamma(\delta)$ and $b=\varphi^{*}\left(q_{0}^{\prime}\right)$. Since $d\left(x_{1}, q_{0}^{\prime}\right)<2 \delta$ and $\operatorname{dim} M_{\varphi}<n$, we can take a point $q_{1}$ in the set

$$
\left\{\exp v ;|v|<2 \delta, v \in T_{x_{1}} M-\hat{C}_{x_{1}}\left(M_{\varphi}\right)\right\} \cap \operatorname{Int} M^{b}\left(\varphi^{*}\right) .
$$

Let $\sigma_{1}:\left[0, d_{1}\right] \rightarrow M$ be the unit speed minimizing geodesic segment from $x_{1}$ to $q_{1}$, and let $c_{1}$ be the minimum of $\varphi^{*}$ on the broken geodesic $\hat{\sigma}_{1}=\left(\sigma_{1} \mid\left[\varepsilon, d_{1}\right]\right) \cup \gamma_{1}$, where $\gamma_{1}$ is the minimizing geodesic segment from $q_{1}$ to $q_{0}^{\prime}$. Notice that $c_{1}>\lambda_{1}$ from the choice of $q_{1}$. Applying Lemma 7.1 to $L_{0}, c_{1}$ and $\hat{\sigma}_{1}$ we see that the point $q_{1}^{\prime}:=\sigma_{1}(\varepsilon)$ is contained in a compact set in $B\left(x_{0}, R\right)$ homeomorphic to $L_{0}$. In particular, $q_{1}^{\prime} \in B\left(x_{0}, R\right)$. On the other hand, we have $d\left(x_{0}, q_{1}^{\prime}\right) \geq d\left(x_{0}, x_{1}\right)-d\left(x_{1}, q_{1}^{\prime}\right) \geq \delta-\varepsilon$. 
Repeating the above argument for $x_{2}=\gamma(2 \delta)$, we get a point $q_{2}^{\prime}$ in $B(x, R)$ with $d\left(x_{0}, q_{2}^{\prime}\right) \geq 2 \delta-\varepsilon$. Continuing this, we see that for every positive integer $k$, there is $q_{k}^{\prime}$ in $B\left(x_{0}, R\right)$ with $d\left(x_{0}, q_{k}^{\prime}\right) \geq k \delta-\varepsilon$. This is a contradiction.

LEMMA 7.3. Suppose that for some $a>\lambda_{1}, \partial M^{a}(\varphi)$ is disconnected and has a compact connected component $L$. If $\sigma:[0,1] \rightarrow M^{a}\left(\varphi^{*}\right)$ is a continuous curve with $\sigma(0) \in L$ and $\sigma(1) \in \partial M^{a}(\varphi)-L$, then for every $c>\lambda_{1}, \sigma$ intersects both $M_{\varphi}$ and Int $M^{c}\left(\varphi^{*}\right)$. In particular, $M_{\varphi} \neq \varnothing$.

Proof. Put $c_{1}=\inf \left\{\varphi^{*}(\sigma(t)) ; 0 \leq t \leq 1\right\}$ and suppose that $\sigma$ does not intersect $M_{\varphi}$. Since $c_{1}>\lambda_{1}$, for $p$ in Int $M^{c_{1}}\left(\varphi^{*}\right)$ we can consider a generalized gradient vector field $X$ of $\left(\varphi^{*}\right.$, dist $\left._{p}\right)$ supported on $M-\operatorname{Int} M^{c_{1}}\left(\varphi^{*}\right)$. Let $\phi_{x}(t)$ be the integral curve of $X$ with $\phi_{x}(0)=x$. From the open-closedness argument as in Lemma 7.1, it turns out that the curve $\phi_{\sigma(s)}(t), t \leq 0$, intersects $L$ transversally for every $s \in[0,1]$. This is a contradiction, because $\sigma(1) \in \partial M^{a}(\varphi)=L$. This argument also shows that $\sigma$ intersects $\operatorname{Int} M^{c}\left(\varphi^{*}\right)$ for all $c>\lambda_{1}$.

PROOF OF THEOREM B. Let $L$ be a compact component of $\partial M^{a}(\varphi)$.

(1) By Lemmas 7.2 and $7.3, M_{\varphi}$ is nonempty and compact. Hence $\varphi^{*}$ has a minimum.

(2) We consider the following two cases.

Case (I). $M_{\varphi}$ coincides with a compact connected component of $\partial M^{a}(\varphi)$.

Then $M_{\varphi}$ is a compact connected totally geodesic hypersurface of $M$ without boundary. For every $x \in M_{\varphi}$, there is a unique unit normal vector $V(x)$ to $M_{\varphi}$ at $x$ with $V(x) \in C_{x}\left(M^{a}\left(\varphi^{*}\right)\right)$. Since $V$ is a globally defined smooth unit normal vector field over $M_{\varphi}$, the normal bundle of $M_{\varphi}$ is trivial.

Case (II). $M_{\varphi}$ does not coincide with any compact connected component of $\partial M^{a}(\varphi)$

We first prove

ASSERTION 1. $M-M_{\varphi}$ is disconnected.

We can take $x \in L-M_{\varphi}$ and $y \in \partial M^{a}(\varphi)-\left(L \cup M_{\varphi}\right)$. To show that $x$ and $y$ cannot be joined by any continuous curve in $M-M_{\varphi}$, suppose the contrary. Let $\sigma:[0,1] \rightarrow M-M_{\varphi}$ be a continuous curve from $x$ to $y$. Put $c=\inf \left\{\varphi^{*}(\sigma(t)) ; 0 \leq\right.$ $t \leq 1\}$. We observe $\lambda_{1}<c \leq a$. For $p \in \operatorname{Int} M^{c}\left(\varphi^{*}\right)$, let $X$ be a generalized gradient vector field of $\left(\varphi^{*}, \operatorname{dist}_{p}\right)$ supported on $M-\operatorname{Int} M^{c}\left(\varphi^{*}\right)$. For each $s \in[0,1]$, let $\hat{\sigma}(s)$ be the intersection of $\partial M^{c}\left(\varphi^{*}\right)$ and the integral curve $\phi_{\sigma(s)}(t)$ of $X$ with $\phi_{\sigma(s)}(0)=\sigma(s)$. Take $t_{0}, t_{1}$ with $\hat{\sigma}(0)=\phi_{x}\left(t_{0}\right)$ and $\hat{\sigma}(1)=\phi_{y}\left(t_{1}\right)$. It turns out that the curves $\phi_{x}(t)\left(0 \leq t \leq t_{0}\right), \hat{\sigma}$ and $\phi_{y}(t)\left(0 \leq t \leq t_{1}\right)$ form a continuous curve in $M^{a}\left(\varphi^{*}\right)-\operatorname{Int} M^{c}\left(\varphi^{*}\right)$ joining $x$ and $y$. This is impossible from Lemma 7.3.

Now, we see that $M_{\varphi}$ has dimension $n-1, n=\operatorname{dim} M$, because $\operatorname{dim} M_{\varphi} \leq n-1$ by Lemma 3.2 , and because $\operatorname{dim} M_{\varphi} \geq n-1$ by the disconnectivity of $M-M_{\varphi}$.

ASSERTION 2. $M_{\varphi}$ has empty boundary.

Suppose that there is a boundary point $p_{0}$ of $M_{\varphi}$. We show that this yields the connectivity of $M-M_{\varphi}$, which is a contradiction. For every $x \in M-M_{\varphi}$, take $p \in M_{\varphi}$ with $d(x, p)=d\left(x, M_{\varphi}\right)$. Let $v \in \nu_{p}\left(M_{\varphi}\right)$ be the unit normal vector to $M_{\varphi}$ at $p$ tangent to a minimizing geodesic from $p$ to $x$. The vector $v$ is joined to a vector $v^{\prime}$ perpendicular to $\hat{C}_{p}\left(M_{\varphi}\right)$ by a curve $V_{t}$ in the set of unit vectors in $\nu_{p}\left(M_{\varphi}\right)$ as follows: If $p \in \operatorname{Int} M_{\varphi}$, we set $V_{t}=v$ since $v$ is perpendicular to $\hat{C}_{p}\left(M_{\varphi}\right)$. If $p \in \partial M_{\varphi}$, we only note that the set $\left\{u \in \nu_{p}\left(M_{\varphi}\right) ;|u|=1\right\}$ is connected, which 
is easily verified. For a fixed $x_{0} \in \operatorname{Int} M_{\varphi}$, let $\gamma:[0,1] \rightarrow M$ be a broken geodesic in $M_{\varphi}$ from $p$ to $p_{0}$ with the break point $x_{0}$. Let $W_{t}, 0 \leq t \leq 1$, be the parallel translate of $v^{\prime}$ along $\gamma$. Since Int $M_{\varphi}$ is totally geodesic and since $\gamma$ lies in Int $M_{\varphi}$, except $p$ and $p_{0}$, we see $W_{t} \in \nu_{\gamma(t)}\left(M_{\varphi}\right)$ for all $t$. Now for a given unit vector $w \in \nu_{p_{0}}\left(M_{\varphi}\right)$, let $Z_{t}$ be a curve in $\nu_{p_{0}}\left(M_{\varphi}\right)$ joining $W_{1}$ and $w$ with $\left|Z_{t}\right|=1$. Take an $\varepsilon>0$ such that $T_{\varepsilon}\left(M_{\varphi}\right)$ is included in a tubular neighborhood of $M_{\varphi}$. The curves $\exp \varepsilon V_{t}, \exp \varepsilon W_{t}$ and $\exp \varepsilon Z_{t}$ form a continuous curve in $M-M_{\varphi}$ joining $\exp \varepsilon v$ and $\exp \varepsilon w$. Thus $x$ and $\exp \varepsilon w$ are joined by a continuous curve in $M-M_{\varphi}$, in an obvious way. Hence $M-M_{\varphi}$ is connected.

The total convexity of $M_{\varphi}$ follows from Proposition 3.9.

The disconnectivity of $M-M_{\varphi}$ also implies that the normal bundle of $M_{\varphi}$ is trivial.

(3) Since $\nu\left(M_{\varphi}\right)$ is trivial, Theorem A shows that $M$ is diffeomorphic to the product $M_{\varphi} \times R$. To prove that $L$ is homeomorphic to $M_{\varphi}$, we first obtain

ASSERTION 3. $L=M_{\varphi}$, or $L \cap M_{\varphi}=\varnothing$.

It suffices to show that if there is $x \in L \cap M_{\varphi}$, then $L=M_{\varphi}$. In fact, similar to the proof of Proposition 3.9, we can conclude $M_{\varphi} \subset \partial M^{a}\left(\varphi^{*}\right)$ and $M_{\varphi}=L$.

Now we may assume $L \cap M_{\varphi}=\varnothing$. We proceed as in the proof of Theorem A. Let $U$ be a tubular neighborhood of $M_{\varphi}$ with $\bar{U} \cap L=\varnothing$. From Lemma 3.7, there is $c>\lambda_{1}$ such that $M^{c}\left(\varphi^{*}\right)$ is included in an $\varepsilon$-tubular neighborhood $U_{1}$ of $M_{\varphi}$ with $\bar{U}_{1} \subset U$. Let $T$ be the vector field on $U-M_{\varphi}$ such that $T(x)$ is tangent to the minimizing geodesic from $x$ to $M_{\varphi}$. For $p \in \operatorname{Int} M^{c}\left(\varphi^{*}\right)$, let $X$ be a generalized gradient vector field of $\left(\varphi^{*}\right.$, dist $\left._{p}\right)$ supported on $M-\operatorname{Int} M^{c}\left(\varphi^{*}\right)$ so that $\langle X, T\rangle(x)>0$ for all $x$ in $\partial U_{1}$. The integral curve of $X$ issuing from each point of $L$ meets $\partial U_{1}$ at a single point. This gives rise to a homeomorphism of $L$ onto a connected component of $\partial U_{1}$, which is homeomorphic to $M_{\varphi}$. We have concluded the proof of Theorem $B$.

REMARK 7.4. Under the situation in Theorem B, if $\varphi$ is lower semicontinuous and quasiconvex, $M_{\varphi}$ coincides with the minimum set of $\varphi$ because a compact Riemannian manifold cannot possess any proper closed totally convex set (see [2, Corollary 1]). Hence $\varphi$ must be constant on the compact totally geodesic submanifold $M_{\varphi}$.

Proof OF TheOREM C. (1) Since $\varphi^{*}$ has no minimum, Lemma 7.2 implies that $M_{\varphi}$ is empty, that is, $\operatorname{dim} M^{c}(\varphi)=n$ for every $c \in\left(\inf _{M} \varphi, a\right]$. Moreover, Lemma 7.1 shows that $\partial M^{c}(\varphi)$ has a compact connected component. From Theorem B, we have that $\partial M^{c}(\varphi)$ is connected.

(2) We note that $M^{c}\left(\varphi^{*}\right)$ is unbounded for every $c \in \varphi^{*}(M)$. For a tubular neighborhood $U$ of $M^{a}\left(\varphi^{*}\right)$, take $\varepsilon>0$ with $T_{\varepsilon}\left(M^{a}\left(\varphi^{*}\right)\right) \subset U$. Since the function $x \mapsto d\left(x, M^{a}\left(\varphi^{*}\right)\right)$ on $U-M^{a}\left(\varphi^{*}\right)$ is of class $C^{1}$, the boundary $L$ of $T_{\varepsilon}\left(M^{a}\left(\varphi^{*}\right)\right)$ is a compact $C^{1}$ hypersurface of $M$. We have to prove that $L$ is homeomorphic to $\partial M^{a}\left(\varphi^{*}\right)$ and $M$ is $C^{1}$ diffeomorphic to $L \times R$. For each nonnegative integer $i$, choose $c_{i} \in \varphi^{*}(M)$ and $p_{i} \in M$ so that $a=c_{0}>\varphi^{*}\left(p_{0}\right)>c_{1}>\varphi^{*}\left(p_{1}\right)>$ $c_{2}>\cdots, \lim c_{i}=\inf _{M} \varphi^{*}$ and $p_{i} \in \operatorname{Int} M^{c_{i}}\left(\varphi^{*}\right)$. Take generalized gradient vector fields $X_{i}$ of $\left(\varphi^{*}, \operatorname{dist}_{\left\{p_{i}, p_{i+1}\right\}}\right)$ supported on $M-\operatorname{Int} M^{c_{i}}\left(\varphi^{*}\right)$. By Lemma 6.1 we may assume that $\left\langle X_{0}(x), T(x)\right\rangle>0$ for all $x \in L$, where $T(x)$ is the unit vector at $x$ tangent to the minimizing geodesic from $x$ to $M^{a}\left(\varphi^{*}\right)$. For smooth functions 
$\rho_{i}: M \rightarrow R$ with $0 \leq \rho_{i} \leq 1$ such that

$$
\rho_{i}= \begin{cases}1 & \text { on } M^{c_{i-1}}\left(\varphi^{*}\right) \\ 0 & \text { outside a small neighborhood of } M^{c_{i-1}}\left(\varphi^{*}\right),\end{cases}
$$

$Y:=\sum_{i=0}^{\infty} \rho_{i} X_{i}$ makes sense as a smooth complete vector field over $M$, where $\rho_{0} \equiv 1$. From the construction, each integral curve of $Y$ intersects $L$ and $\partial M^{a}\left(\varphi^{*}\right)$ transversally. This gives rise to a homeomorphism of $L$ onto $\partial M^{a}\left(\varphi^{*}\right)$, a $C^{1}$ diffeomorphism of $M$ onto $L \times R$.

REMARK 7.5. From Theorems B, C and their proofs, we have that for every $a>\lambda_{1}, \partial M^{a}(\varphi)$ has at most two compact connected components:

(1) If $\partial M^{a}(\varphi)$ has exactly two compact connected components $L_{0}$ and $L_{1}$, then $\partial M^{a}(\varphi)=L_{0} \cup L_{1}$ and $\overline{M^{a}(\varphi)}$ is homeomorphic to $L_{0} \times[0,1]$.

(2) If $\partial M^{a}(\varphi)$ consists of a single compact connected component $L_{0}$ and if $M_{\varphi}=\varnothing$, then $\overline{M^{a}(\varphi)}$ is homeomorphic to $L_{0} \times[0, \infty)$.

8. End structure. Let $M$ be a noncompact manifold. If $K_{1}$ and $K_{2}$ are compact subsets of $M$ with $K_{1} \subset K_{2}$, then each connected component of $M-K_{2}$ is included in a unique connected component of $M-K_{1}$. An end of $M$ is by definition an element of the inverse system

\section{\{connected components of $M-K ; K$ compact in $M$ \}}

directed by inclusion and reverse inclusion. Specifically, an end $\varepsilon$ is an assignment to each compact set $K$ in $M$ a connected component $\varepsilon(K)$ of $M-K$ in such a way that $\varepsilon\left(K_{1}\right) \supset \varepsilon\left(K_{2}\right)$ if $K_{1} \subset K_{2}$. Every noncompact manifold has at least one end.

It has been shown in [7] that if a complete Riemannian manifold admits a locally nonconstant convex function, then it has at most two ends. We generalize this result to manifolds admitting locally nonconstant quasiconvex functions.

THEOREM D. If a complete Riemannian manifold $M$ admits a locally nonconstant locally quasiconvex function $\varphi \cdot M \rightarrow R \cup\{+\infty\}$, then $M$ has at most two ends: More precisely,

Case (I). $\partial M^{a}(\varphi)$ has a compact connected component for some a $>\lambda_{1}$.

(1) If $M_{\varphi} \neq \varnothing$ and $\partial M^{c}(\varphi)$ is connected for every $c \in\left(\lambda_{1}, a\right]$, then $M$ has exactly one end.

(2) If $M_{\varphi} \neq \varnothing$ and $\partial M^{c}(\varphi)$ is disconnected for some $c \in\left(\lambda_{1}, a\right]$, then $M$ has exactly two ends.

(3) If $M_{\varphi}=\varnothing$, then $M$ has exactly two ends.

Case (II). For each $a>\lambda_{1}$, every connected component of $\partial M^{a}(\varphi)$ is noncompact.

In this case, $M$ always has exactly one end.

PROOF. (I)(1) From Lemma 7.2, we can take a bounded tubular neighborhood $U$ of $M_{\varphi}$. By Lemma 3.7, there is $c \in\left(\lambda_{1}, a\right]$ with $M^{c}\left(\varphi^{*}\right) \subset U$. In particular, $M^{c}\left(\varphi^{*}\right)$ is compact. On the other hand, $M-\operatorname{Int} M^{c}\left(\varphi^{*}\right)$ is homeomorphic to $\partial M^{c}\left(\varphi^{*}\right) \times[0, \infty)$ by Corollary 5.6. Since $\partial M^{c}\left(\varphi^{*}\right)$ is connected, this observation implies that $M$ has exactly one end.

(2), (3) In these cases, the conclusions have already been shown in Theorems B and $\mathrm{C}$. 
(II) We divide this case into the following two cases.

Case (i). $M_{\varphi}=\varnothing$. Let $K$ be a compact set of $M$. By the lower semicontinuity of $\varphi^{*}$, we can take $a \in \varphi^{*}(M)$ so that $K$ does not meet $M^{a}\left(\varphi^{*}\right)$. For $p \in \operatorname{Int} M^{a}\left(\varphi^{*}\right)$, let $X$ be a generalized gradient vector field of $\left(\varphi^{*}, \operatorname{dist}_{p}\right)$ supported on $M-$ Int $M^{a}\left(\varphi^{*}\right)$. Let $U$ be an unbounded connected component of $M-K$. Since $M^{a}\left(\varphi^{*}\right)$ is connected, we observe that $U \supset M^{a}\left(\varphi^{*}\right)$ or $U \cap M^{a}\left(\varphi^{*}\right)=\varnothing$. Suppose $U \cap M^{a}\left(\varphi^{*}\right)=\varnothing$. Then there is $q \in U$ such that the integral curve $\phi_{q}(t), t \leq 0$, of $X$ with $\phi_{q}(0)=q$ does not intersect $K$. Let $P: M-\operatorname{Int} M^{a}\left(\varphi^{*}\right) \rightarrow \partial M^{a}\left(\varphi^{*}\right)$ be the projection onto $\partial M^{a}\left(\varphi^{*}\right)$ via the integral curves of $X$. Since the connected component $L$ of $\partial M^{a}\left(\varphi^{*}\right)$ containing $P(q)$ is noncompact, we can take $q^{\prime} \in L-P(K)$. Let $\sigma$ be a continuous curve in $L$ joining $P(q)$ and $q^{\prime}$. It turns out that for large $t>0$, the curve $s \mapsto \phi_{\sigma(s)}(-t)$ joins $\phi_{P(q)}(-t)$ and $\phi_{q^{\prime}}(-t)$ in $M-K$. Thus $q$ and $q^{\prime}$ are also joined in $M-K$ in an obvious way. Hence $q^{\prime} \in U$ and $U \cap M^{a}\left(\varphi^{*}\right) \neq \varnothing$. This is a contradiction. Therefore $U$ is the only unbounded connected component of $M-K$ with $U \supset M^{a}\left(\varphi^{*}\right)$, and $M$ has exactly one end.

Case (ii). $M_{\varphi} \neq \varnothing$. We note that $M_{\varphi}$ is unbounded by Lemma 3.7. Let $F: M-M_{\varphi} \rightarrow L \times(0, \infty)$ be the $C^{1}$ diffeomorphism given in Theorem $\mathrm{A}$, where $L$ is a $C^{1}$ hypersurface of $M$. Note that $F^{-1}(x, t)$ goes to infinity as $t \rightarrow+\infty$ for each $x \in L$, and that $F$ defines the projection pr: $M \rightarrow M_{\varphi}$. For every compact set $K$ of $M$, we can take $p \in M_{\varphi}-\operatorname{pr}(K)$. Let $U$ be an unbounded connected component of $M-K$. We can choose $q \in U$ such that if we set $F(q)=\left(x_{0}, t_{0}\right)$, then $F^{-1}\left(x_{0}, t\right) \notin K$ for all $t \geq t_{0}$. Now similarly to Case (i), the points $p$ and $q$ are joined in $M-K$. Therefore $M$ has exactly one end.

ADDED IN PROOF. In a future paper, we shall show that if $M$ admits a locally nonconstant locally quasiconvex function $\varphi$ with $M_{\varphi}=\varnothing$, then there is a $C^{\infty}$ manifold $N$ such that $M$ is diffeomorphic to $N \times R$.

\section{REFERENCES}

1. V. Bangert, Riemannsche Mannigfaltigkeiten mit nicht-konstanten konvexer Funktionen, Arch. Math. 31 (1978), 163-170.

2. (1981), 333-345.

3. R. L. Bishop and B. O'Neill, Manifolds of negative curvature, Trans. Amer. Math. Soc. 145 (1969), 1-49.

4. Yu. D. Burago and V. A. Zalgaller, Convex sets in Riemannian spaces of non-negative curvature, Russian Math. Surveys 32 (3) (1977), 1-57.

5. H. Busemann and B. B. Phadke, Peakless and monotone functions on $G$-spaces, Tsukuba J. Math. 7 (1983), 105-135.

6. J. Cheeger and G. Gromoll, On the structure of complete manifolds of nonnegative curvature, Ann. of Math. (2) 96 (1972), 413-443.

7. R. E. Greene and K. Shiohama, Convex functions on complete noncompact manifolds: Topological structure, Invent. Math. 63 (1981), 129-157.

8. - Convex functions on complete noncompact manifolds: Differentiable structure, Ann. Sci. École Norm. Sup. 14 (1981), 357-367.

9. __ The isometry groups of manifolds admitting nonconstant convex functions (preprint).

10. R. E. Greene and $\mathrm{H}$. Wu, $C^{\infty}$ convex functions and manifolds of positive curvature, Acta Math. 137 (1976), 209-245.

11. D. Gromoll and W. Meyer, On complete open manifolds of positive curvature, Ann. of Math (2) 90 (1969), 75-90. 
12. M. Gromov, Curvature, diameter and Betti numbers, Comment. Math. Helv. 56 (1981), 179-195.

13. R. Walter, On the metric projection onto convex sets in Riemannian spaces, Arch. Math. 25 (1974), 91-98.

14. $\mathrm{T}$. Yamaguchi, The isometry groups of Riemannian manifolds admitting strictly convex functions, Ann. Sci. École Norm. Sup. (4) 15 (1982), 205-212.

15. S. T. Yau, Nonexistence of continuous convex functions on certain Riemannian manifolds, Math. Ann. 207 (1974), 269-270.

Department of Mathematics, Faculty of Science and Engineering, Saga UNIVERSITY, SAGA 840, JAPAN 